\title{
ESO VERY LARGE TELESCOPE OPTICAL SPECTROSCOPY OF BL LACERTAE OBJECTS. II. NEW REDSHIFTS, FEATURELESS OBJECTS, AND CLASSIFICATION ASSESSMENTS
}

\author{
B. Sbarufatti ${ }^{1}$ and A. TReves \\ Università dell'Insubria, Via Valleggio 11, I-22100 Como, Italy \\ R. FALOMO \\ INAF-Osservatorio Astronomico di Padova, Vicolo dell'Osservatorio 5, I-35122 Padua, Italy \\ J. HEIDT \\ Landenssterwarte Heidelberg, Königstuhl, D-69117 Heidelberg, Germany \\ J. Kotilainen \\ Tuorla Observatory, University of Turku, Väisäläntie 20, FIN-21500 Piikkiö, Finland \\ AND \\ R. SCARPA \\ European Southern Observatory, 3107 Alonso de Cordova, Santiago, Chile \\ Received 2005 July 29; accepted 2006 January 28
}

\begin{abstract}
We report on ESO Very Large Telescope optical spectroscopy of 42 BL Lacertae objects of unknown redshift. Nuclear emission lines were observed in 12 objects, while for another six we detected absorption features due to the host galaxy. The new high signal-to-noise ratio spectra therefore allow us to measure the redshift of 18 sources. Five of the observed objects were reclassified as either stars or quasars, and one is of uncertain nature. For the remaining 18 the optical spectra appear without intrinsic features in spite of our ability to measure rather faint (equivalent width $\sim 0.1 \AA$ ) spectral lines. For the latter sources a lower limit to the redshift was set, exploiting the very fact that the absorption lines of the host galaxy are undetected in the observed spectra.
\end{abstract}

Key words: BL Lacertae objects: general — galaxies: distances and redshifts

\section{INTRODUCTION}

BL Lac objects (BLLs) are active galactic nuclei (AGNs) characterized by luminous, rapidly variable UV-to-near-IR nonthermal continuum emission and polarization, strong compact flat-spectrum radio emission, and superluminal motion. Similar properties are also observed in flat-spectrum radio quasars (FSRQ), and the two types of active nuclei are often grouped together in the blazar class. From the spectroscopic point of view BLLs are characterized by quasi-featureless optical spectra. In fact, their spectra are often dominated by the nonthermal continuum that arises from the nucleus. On this emission is superposed a thermal contribution due to the stellar component of the host galaxy. As in other AGNs, emission lines could be generated by fluorescence in clouds surrounding the central black hole. Moreover, as happens for high- $z$ quasars, in some cases absorption lines due to intervening gas in the halo of foreground galaxies can be observed in the spectra of BLLs, and one can derive a lower limit to the redshift of the object. The detectability of spectral features depends on the brightness of the nuclear source; in fact, during low brightness states, intrinsic absorption features can be more easily revealed, while during high states one can better discover intervening absorption systems. Because of the strong contribution from the continuum, the equivalent width (EW) of all these spectral features is often very small, and their detection represents a challenging task.

In the past decade a number of projects were carried out to derive the redshift of BLLs. Most of these works were based on optical spectra collected with $4 \mathrm{~m}$ class telescopes and are

\footnotetext{
${ }^{1}$ Also at Università degli Studi di Milano-Bicocca, I-20133 Milan, Italy.
}

therefore limited by relatively low signal-to-noise ratio $(\mathrm{S} / \mathrm{N})$, low spectral resolution, and limited wavelength range (e.g., Falomo et al. 1993, 1994; Stickel \& Kühr 1993; Véron-Cetty \& Véron 1993b; Bade et al. 1994; Falomo 1996; Marchã et al. 1996; Drinkwater et al. 1997; Laurent-Muehleisen et al. 1998; Landt et al. 2001; Rector \& Stocke 2001; Londish et al. 2002; Carangelo et al. 2003; Hook et al. 2003). Recently, however, some observations with $8 \mathrm{~m}$ class telescopes have been carried out (Heidt et al. 2004; Sowards-Emmerd et al. 2005). Despite these efforts, a significant fraction of known BLLs (e.g., 50\% in the VéronCetty \& Véron [2003] catalog) still have unknown redshifts.

In order to improve the knowledge of the redshift of BLLs we carried out a project to obtain optical spectra of sources with still unknown or uncertain redshift using the European Southern Observatory (ESO) $8 \mathrm{~m}$ Very Large Telescopes (VLT). This allows one to improve significantly the $\mathrm{S} / \mathrm{N}$ of the spectra and therefore the capability of detecting faint spectral features. A first report on this work, giving the redshifts of 12 objects, has been presented by Sbarufatti et al. (2005b, hereafter Paper I), and here we refer to the results for the full sample of 42 observed sources.

The outline of this paper is the following. In $\S 2$ we give some characterization of the 42 observed objects. The observation and analysis procedures are described in $\S 3$. In $\S \S 4$ and 5 we report the results of our spectroscopic study. Finally, in $\S 6$ a summary and conclusions of this study are given. Throughout this paper we adopt the following cosmological parameters: $H_{0}=70 \mathrm{~km}$ $\mathrm{s}^{-1} \mathrm{Mpc}^{-1}, \Omega_{\Lambda}=0.7$, and $\Omega_{m}=0.3$.

\section{THE SAMPLE}

The sample of BLLs (and candidates) observed with the VLT was selected from two extended lists of BLLs: the Padovani \& 


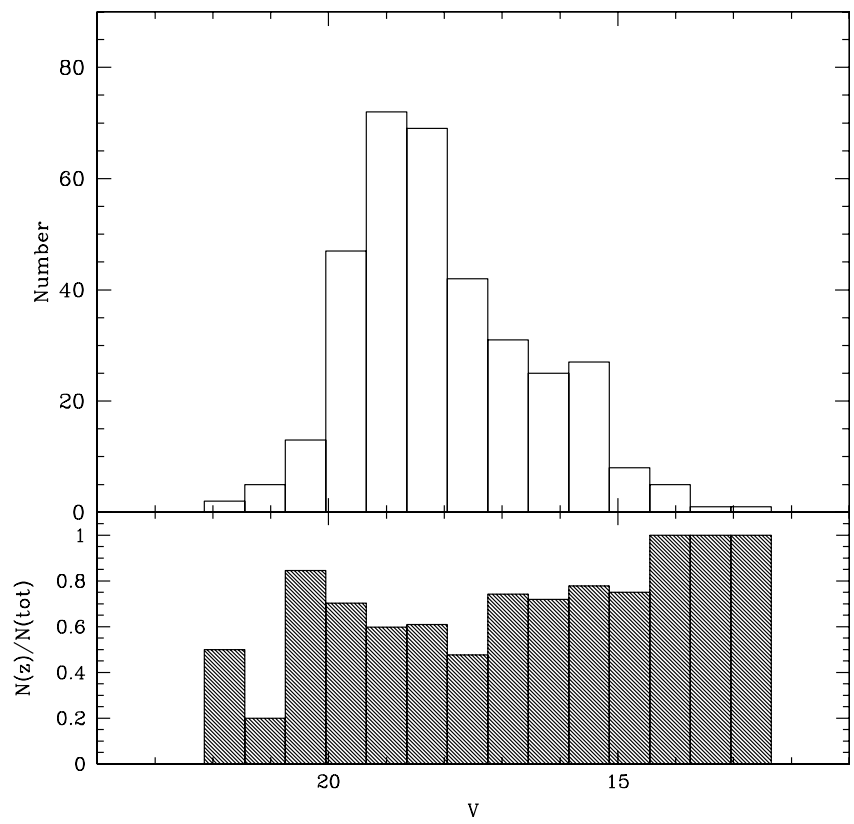

FIg. 1.-Top: $V$-magnitude distribution of BLLs from Padovani \& Giommi (1995a) and SS. Bottom: Fraction of objects of known redshift as a function of the magnitude.
Giommi (1995a) collection of BLLs and the Sedentary Survey (SS; Giommi et al. 1999, 2005). The Padovani \& Giommi (1995a) list contains all objects identified as BLLs belonging to the complete samples existing at the time of its compilation, selected in the radio, optical, and X-ray bands (e.g., the 1 Jy survey, Stickel et al. 1991; Palomar-Green survey [PG], Green et al. 1986; Extended Medium Sensitivity Survey [EMSS], Gioia et al. 1990; Slew Survey, Perlman et al. 1996; and the White-GiommiAngelini catalog, White et al. 1994). It also includes sources from the Hewitt \& Burbidge (1993) and Véron-Cetty \& Véron (1993a) catalogs (in the latter case we checked that the source was still included in the 2001 version), for a total of 233 objects. The criteria used to define a BL Lac object in Padovani \& Giommi (1995a) depend on the original sample. In most cases, the EW of the lines is required to be $\leq 5 \AA$, but UV excess, optical polarization and variability, and radio-to-optical spectral index are also used as selection criteria. The SS was obtained by crosscorrelating the NRAO VLA Sky Survey (NVSS) data (Condon et al. 1998) with the RASS Bright Source Catalogue (RBSC) list of sources (Voges et al. 1999). SS selected a complete sample of 150 high-energy peaked BLLs (see Padovani \& Giommi [1995b] for a definition) down to a $3.5 \mathrm{mJy}$ radio flux limit. BL Lac classification in the SS is based on the position of the sources on the $\alpha_{\mathrm{ox}}-\alpha_{\text {ro }}$ plane.

TABLE 1

Journal of Observations and Results for Objects Not Reported in Paper I

\begin{tabular}{|c|c|c|c|c|c|c|c|c|c|c|}
\hline $\begin{array}{c}\text { Object Name } \\
\text { (1) }\end{array}$ & $\begin{array}{l}\text { IAU Name } \\
\text { (2) }\end{array}$ & $\begin{array}{c}\text { R.A. } \\
(\mathrm{J} 2000.0) \\
(3)\end{array}$ & $\begin{array}{c}\text { Decl. } \\
(\mathrm{J} 2000.0) \\
(4)\end{array}$ & $\begin{array}{l}\text { Date } \\
(5)\end{array}$ & $\begin{array}{l}t_{\mathrm{exp}} \\
(6)\end{array}$ & $\begin{array}{l}\mathrm{S} / \mathrm{N} \\
(7)\end{array}$ & $\begin{array}{c}R \\
(8)\end{array}$ & $\begin{array}{l}\alpha \\
(9)\end{array}$ & $\begin{array}{c}\mathrm{EW}_{\text {min }} \\
(10)\end{array}$ & $\begin{array}{c}z \\
(11)\end{array}$ \\
\hline PKS $0047+023 \ldots$. & $0047+023$ & 004943.2 & +023704.8 & 2003 Aug 5 & 1800 & 80 & 19.0 & 0.61 & 0.36 & $>0.82$ \\
\hline PKS $0048-09$. & $0048-097$ & 005041.3 & -09 2905.2 & 2003 Sep 17 & 1800 & 250 & 16.0 & 0.95 & 0.22 & $>0.30$ \\
\hline PKS $0420+022$. & $0420+022$ & 042252.2 & +021926.9 & 2003 Nov 19 & 2325 & 90 & 18.9 & . & 0.41 & 2.278 \\
\hline PKS $0422+00 \ldots$ & $0422+004$ & 042446.8 & +003606.3 & 2003 Nov 27 & 2325 & 230 & 16.2 & 0.88 & 0.25 & $>0.31$ \\
\hline PKS $0627-199 \ldots$ & $0627-199$ & 062923.8 & -195919.7 & 2003 Dec 16 & 2325 & 50 & 19.3 & 0.56 & 0.92 & $>0.63$ \\
\hline PKS 0723-00 & $0723-008$ & 072550.6 & -005456.5 & 2003 Dec 25 & 2325 & 250 & 16.0 & $\ldots$ & 0.23 & 0.127 \\
\hline $\mathrm{H} 0841+1256 \ldots \ldots \ldots \ldots$. & $0841+129$ & 084424.1 & +124548.0 & 2003 Dec 30 & 2325 & 100 & 18.0 & $\ldots$ & 0.38 & $>2.48$ \\
\hline 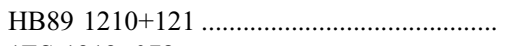 & $1210+121$ & 121233.9 & +115056.9 & 2004 Jan 24 & 2325 & 180 & 17.8 & $\ldots$ & 0.28 & $\ldots$ \\
\hline 1ES $1212+078 \ldots$. & $1212+078$ & 121510.9 & +073203.8 & 2004 Jan 25 & 2325 & 100 & 17.3 & 1.17 & 0.39 & 0.137 \\
\hline $1222+102 \ldots \ldots \ldots \ldots$ & $1222+102$ & 122523.1 & +095935.0 & 2004 Jan 26 & 2325 & 160 & 17.7 & 2.67 & 0.30 & $\ldots$ \\
\hline 1ES $1248-296$ & $1248-296$ & 125134.9 & -295842.9 & 2004 Jan 24 & 2325 & 50 & 19.5 & 0.92 & 0.57 & 0.382 \\
\hline 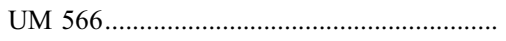 & $1319+019$ & 131955.1 & +015258.3 & 2003 Apr 30 & 1800 & 100 & 18.2 & $\ldots$ & 0.36 & \\
\hline $1 \mathrm{ES} 1320+084 \mathrm{~N}$. & $1320+084$ & 132254.9 & +081010.0 & 2003 Apr 30 & 2325 & 50 & 19.5 & $\ldots$ & 0.54 & 1.500 \\
\hline PKS $1349-439 .$. & $1349-439$ & 135256.5 & -441240.4 & 2003 Apr 30 & 1800 & 240 & 16.9 & 0.82 & 0.32 & $>0.39$ \\
\hline 1RXS J144505.9-032613. & $1442-032$ & 144505.8 & -032612.8 & 2004 Aug 28 & 2325 & 100 & 17.7 & 1.21 & 0.35 & $>0.51$ \\
\hline 1RXS J150343.0-154107.. & $1500-154$ & 150342.9 & -154107.0 & 2004 Aug 28 & 2325 & 40 & 17.8 & 1.52 & 0.78 & $>0.38$ \\
\hline 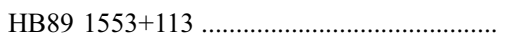 & $1553+113$ & 155543.0 & +111124.4 & 2003 Aug 1 & 1800 & 250 & 14.0 & 0.84 & 0.25 & $>0.09$ \\
\hline $\mathrm{H} 1722+119 \ldots \ldots \ldots$ & $1722+119$ & 172504.4 & +115215.2 & 2003 Apr 6 & 1800 & 350 & 14.7 & 1.30 & 0.18 & $>0.17$ \\
\hline PKS 2012-017 ................... & $2012-017$ & 201515.2 & -013733.0 & 2003 Jul 31 & 1800 & 130 & 19.3 & 0.49 & 0.34 & $>0.94$ \\
\hline 1RXS J213151.7-251602.. & $2128-254$ & 213151.6 & -251600.8 & 2004 Jul 10 & 2325 & 70 & 19.0 & 1.28 & 0.32 & $>0.86$ \\
\hline 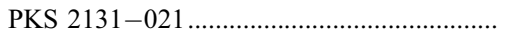 & $2131-021$ & 213410.3 & -015317.0 & 2004 Jul 18 & 2325 & 80 & 19.2 & 0.29 & 0.43 & 1.284 \\
\hline 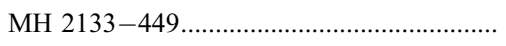 & $2133-449$ & 213618.4 & -444349.0 & 2004 Jul 12 & 2325 & 60 & 19.5 & 1.02 & 0.37 & $>0.98$ \\
\hline MH 2136-428 & $2136-428$ & 213924.1 & -423521.3 & 2003 Jul 3 & 1800 & 490 & 15.6 & 0.84 & 0.24 & $>0.24$ \\
\hline 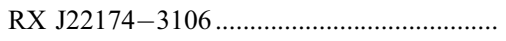 & $2214-313$ & 221728.4 & -310619.0 & 2004 Jul 10 & 2325 & 50 & 19.7 & 0.90 & 0.68 & 0.460 \\
\hline 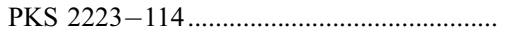 & $2223-114$ & 222543.6 & -111340.0 & 2004 Sep 2 & 2325 & 20 & 21.5 & 0.31 & 1.04 & 0.997 \\
\hline PKS 2233-148 & $2233-148$ & 223634.0 & -143321.0 & 2004 Sep 2 & 2325 & 170 & 18.5 & 0.15 & 0.30 & $>0.65$ \\
\hline PKS 2254-204 & $2254-204$ & 225641.2 & -201140.3 & $2003 \mathrm{Jul} 31$ & 1800 & 220 & 17.1 & 0.86 & 0.25 & $>0.47$ \\
\hline 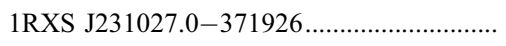 & $2307-375$ & 231026.9 & -371926.0 & 2004 Jul 10 & 2325 & 80 & 19.6 & 1.15 & 0.34 & $>1.03$ \\
\hline 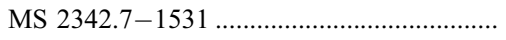 & $2342-153$ & 234522.4 & -151506.7 & 2003 Jul 26 & 2325 & 20 & 21.4 & 1.02 & 1.72 & $>1.03$ \\
\hline 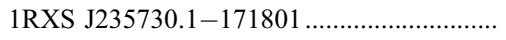 & $2354-175$ & 235729.7 & -171805.3 & 2004 Jul 12 & 2325 & 150 & 18.2 & 1.44 & 0.17 & $>0.85$ \\
\hline
\end{tabular}

Notes-Units of right ascension are hours, minutes, and seconds, and units of declination are degrees, arcminutes, and arcseconds. Col. (1): Object name. Col. (2): IAU name. Col. (3): Right ascension (J2000.0). Col. (4): Declination (J2000.0). Col. (5): Date of observations. Col. (6): Exposure time (s). Col. (7): Signal-to-noise ratio. Col. (8): $R$-band magnitude. Col. (9): Spectral index of the continuum, $\alpha$, defined by $F_{\lambda} \propto \lambda^{-\alpha}$. Col. (10): Minimum detectable equivalent width. Col. (11): Redshifts measured from spectral features and redshift lower limits from the procedure described in $\S 4.2 .3$. 

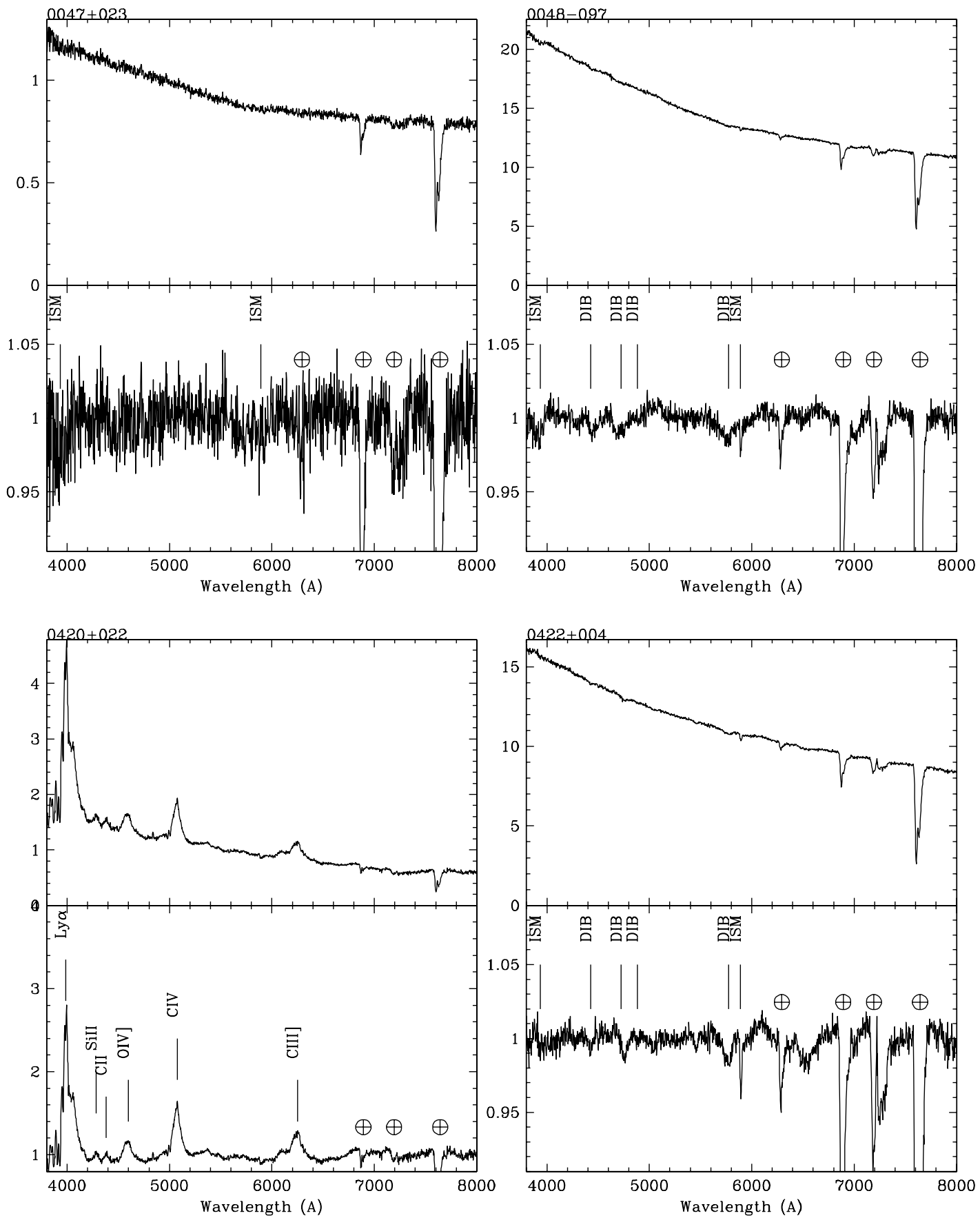

FIG. 2.-Spectra of the observed objects. Top panels: Flux-calibrated dereddened spectra. Bottom panels: Normalized spectra. Telluric bands are indicated by the Earth symbol, spectral lines are marked by the line identification, intervening $\mathrm{Mg}$ II absorption systems are reported as "int. Mg II," unidentified intervening systems are indicated with an asterisk, absorption features from atomic species in the interstellar medium of our Galaxy are labeled by ISM, and diffuse interstellar bands are identified by DIB.

The Padovani \& Giommi (1995a) and SS data sets lead to a combined list containing 348 objects. The distribution of the $V$ magnitude for these objects is shown in Figure 1. The bulk of them have $V$ between 15 and 20, and the fraction of objects with unknown redshift increases with the apparent magnitude and reaches $\sim 50 \%$ at the faintest magnitudes. Note, however, that also at $V \sim 15-17$ about $20 \%$ of the sources have no known redshift. The total number of objects with unknown redshifts is 105 .

From the combined list we selected sources with $\delta<+15^{\circ}$ for observability from the VLT site. Moreover, to grant a sufficiently high S/N level of the optical spectra, we required $V<22$. Thus we gathered a list of 59 objects. During three observational campaigns, performed in service mode, we completed this optical spectroscopy program, obtaining data for $\sim 70 \%$ of the sample (42 sources). Our sample is similar to the parent sample of 348 objects in terms of mean apparent magnitude and subdivision in low- and high-energy peaked BLLs.

\section{OBSERVATIONS AND DATA ANALYSIS}

Optical spectra were collected in service mode with FORS1 (Appenzeller et al. 1998) on the VLT. The observations were obtained from 2003 April to 2004 March with UT1 (Antu) and from 2004 April to October with UT2 (Kueyen). We used the 

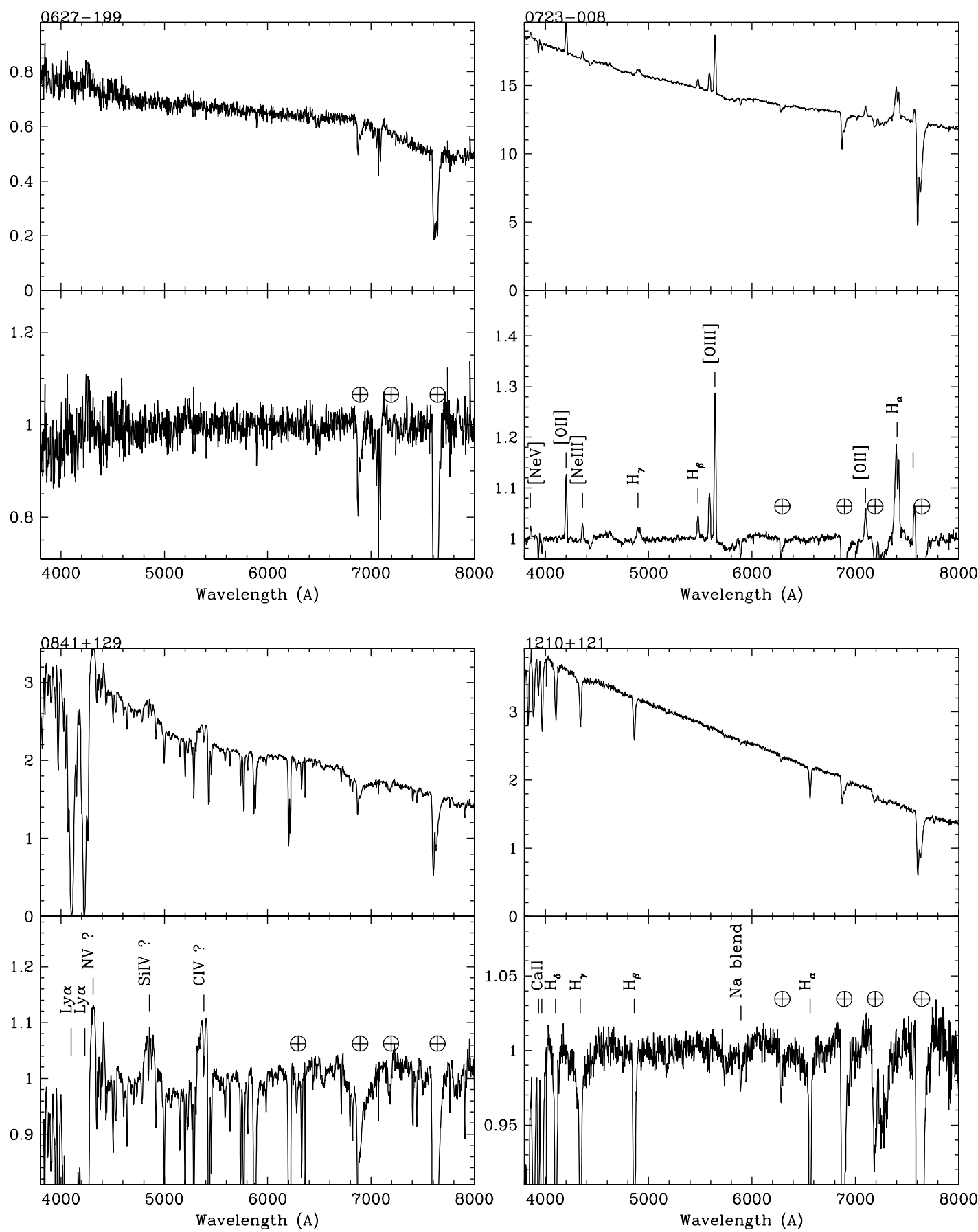

Fig. 2.-Continued

$300 \mathrm{~V}+I$ grism combined with a $2^{\prime \prime}$ slit, yielding a dispersion of $110 \AA \mathrm{mm}^{-1}$ (corresponding to $2.64 \AA \mathrm{pixel}^{-1}$ ) and a spectral resolution of $15-20 \AA$ covering the $3800-8000 \AA$ range. The seeing during observations was in the range $0.5-2.5$, with an average of $\sim 1^{\prime \prime}$. Relevant information on the sample objects is given in Table 1 .

Data reduction was performed using $\operatorname{IRAF}^{2}$ (Tody 1986, 1993) following standard procedures for spectral analysis. This included bias subtraction, flat-fielding, and cleaning for bad pixels. For each target we obtained three spectra in order to get a

\footnotetext{
${ }^{2}$ IRAF (Image Reduction and Analysis Facility) is distributed by the National Optical Astronomy Observatory, which is operated by the Association of Universities for Research in Astronomy, Inc., under cooperative agreement with the National Science Foundation.
}

good correction for cosmic rays and to check the reality of weak features. The individual frames were then combined into a single average image. Wavelength calibration was performed using the spectra of a helium-neon-argon lamp obtained during the same observing night, reaching an accuracy of $\sim 3 \AA$ (rms). From these images we extracted one-dimensional spectra, adopting an optimal extraction algorithm (Horne 1986) to improve the S/N.

Although this program did not require optimal photometric conditions, most of the observations were obtained with a clear sky. This enabled us to perform a spectrophotometric calibration of the acquired data using standard stars (Oke 1990) observed in the same nights. From the database of sky conditions at Paranal we estimate that a photometric accuracy of $10 \%$ was reached during our observing nights. The spectra were also corrected for 

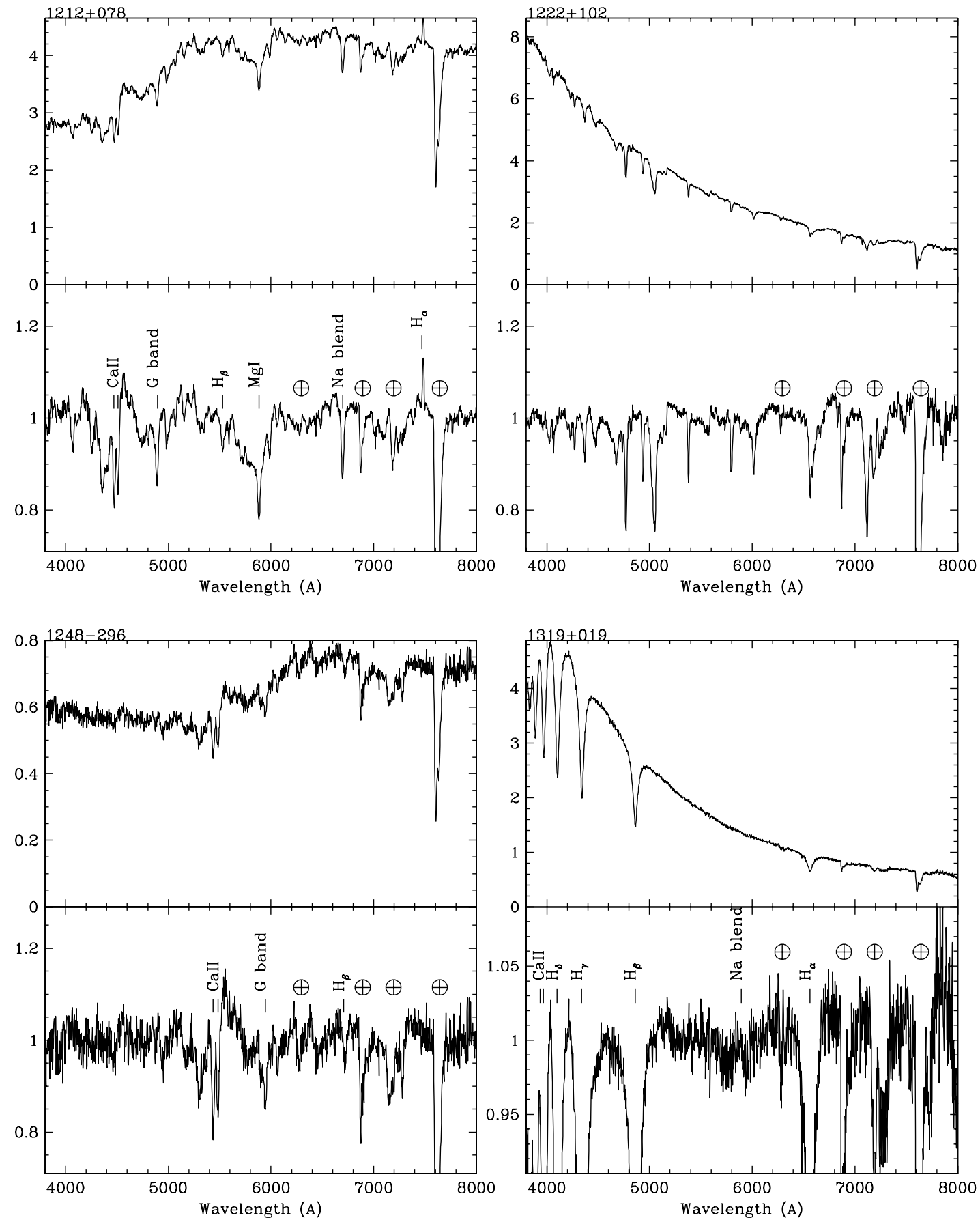

FIG. 2.-Continued

Galactic extinction, using the law of Cardelli et al. (1989) and assuming values of $E(B-V)$ from Schlegel et al. (1998).

\section{RESULTS}

In Figure 2 we give the optical spectrum of each source. In order to show more clearly the continuum shape and the faint features, we report both the flux-calibrated and the normalized spectra for each object. The main emission and absorption features are identified. Those due to the Galactic interstellar gas are indicated as "ISM" (interstellar medium) or "DIB" (diffuse interstellar bands; see $\S 4.2 .3$ ), while telluric absorptions are marked with an Earth symbol. ${ }^{3}$

\footnotetext{
${ }^{3}$ All spectra of BL Lac objects can be retrieved at http://www.oapd.inaf.it.
}

\subsection{The Continuum Emission}

In a first approximation, the optical continuum of a BL Lac object is due to the superposition of two components: the nonthermal emission of the active nucleus, Doppler-enhanced because of the alignment of the jet with the line of sight, and the emission of the host galaxy. Depending on the relative strength of the nucleus with respect to the galaxy light, the spectral signature of the latter can be either easily detected or diluted beyond the point of recognition. Taking into account the robust evidence that the hosts are giant elliptical galaxiess (e.g., Urry et al. 2000), we fitted a power law $\left(F_{\lambda} \propto \lambda^{-\alpha}\right.$; the spectral indices are given in Table 1) plus the spectrum of a typical elliptical galaxy, as described by the Kinney et al. (1996) template, to describe the continuum and derive the 

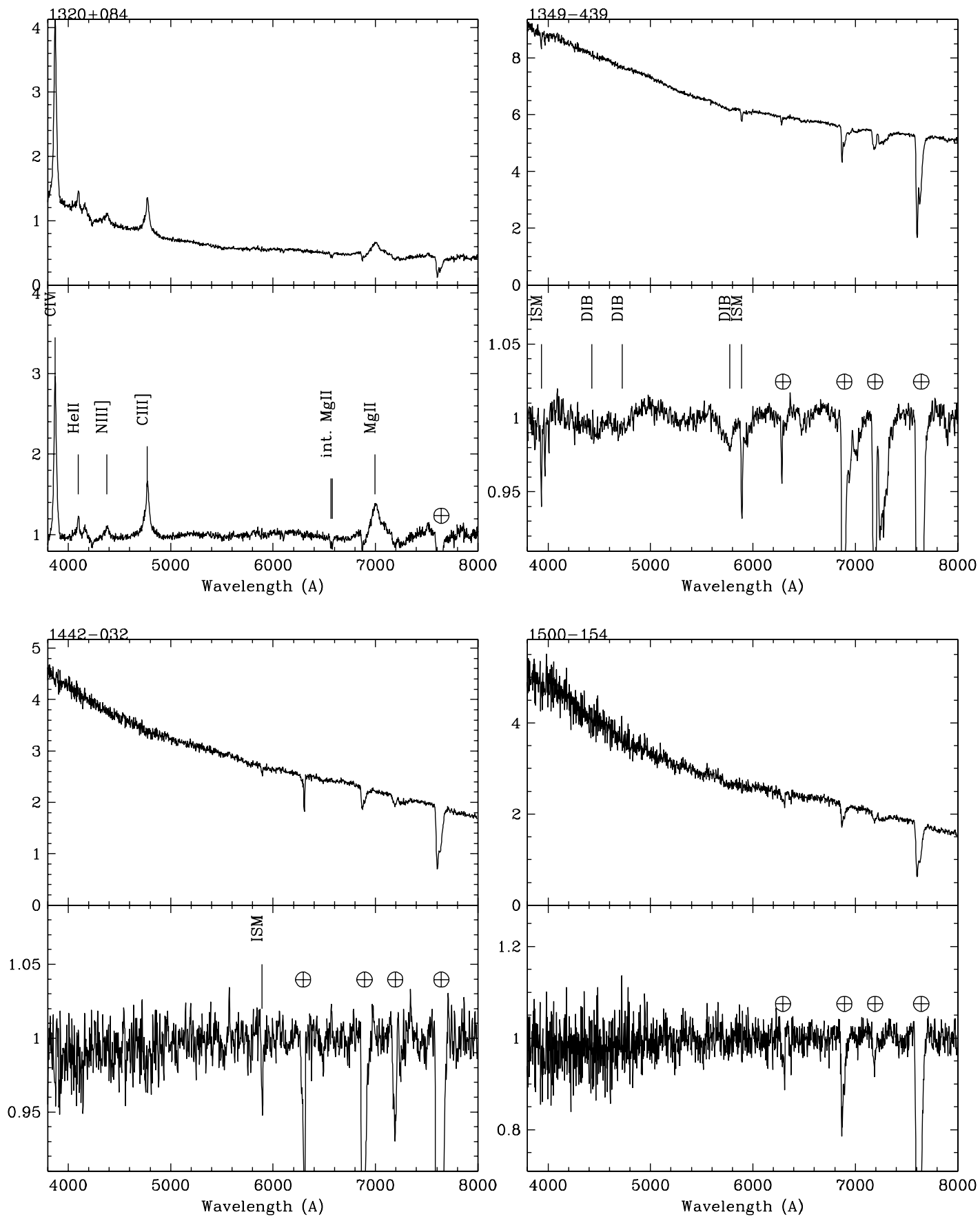

Fig. 2.-Continued

optical spectral index of the nonthermal component. While in most cases the contribution of the host galaxy was negligible, in six sources it was not, and the luminosity of the host could thus be derived. For these six sources (three of which were presented in Paper I) we give the best-fit decomposition in Figure 3 and report the parameters in Table 2. The derived absolute magnitudes of the host galaxies are consistent with the distribution of $M_{R}$ of BL Lac hosts given by Sbarufatti et al. (2005a).

\subsection{Spectral Features and Redshifts}

The detection and the measurement of very weak spectral features is difficult to assess because it depends on the choice of the parameters used to define the spectral line and the continuum. In order to apply an objective method for any given spectrum we evaluate the minimum measurable equivalent width $\left(\mathrm{EW}_{\min }\right)$, defined as twice the rms of the distribution of all equivalent width values measured dividing the normalized spectrum into $30 \AA$ wide bins (details for this automatic routine are given in Paper I). We checked that the $\mathrm{S} / \mathrm{N}$ dependence inside the considered spectral range varies at most by $20 \%$, remaining $<10 \%$ over a large wavelength range. This is reflected in a similar variation of $\mathrm{EW}_{\min }$. The procedure for calculating $\mathrm{EW}_{\min }$ was applied to all featureless or quasi-featureless spectra to find faint spectral lines. All features above the $\mathrm{EW}_{\min }$ threshold, ranging from $\sim 1$ to $0.1 \AA$ in our data, were considered as line candidates and were carefully visually inspected and measured. The results are summarized in Table 3. Based on the detected lines and the shape of the continuum we confirm the BL Lac classification for 36 objects, 

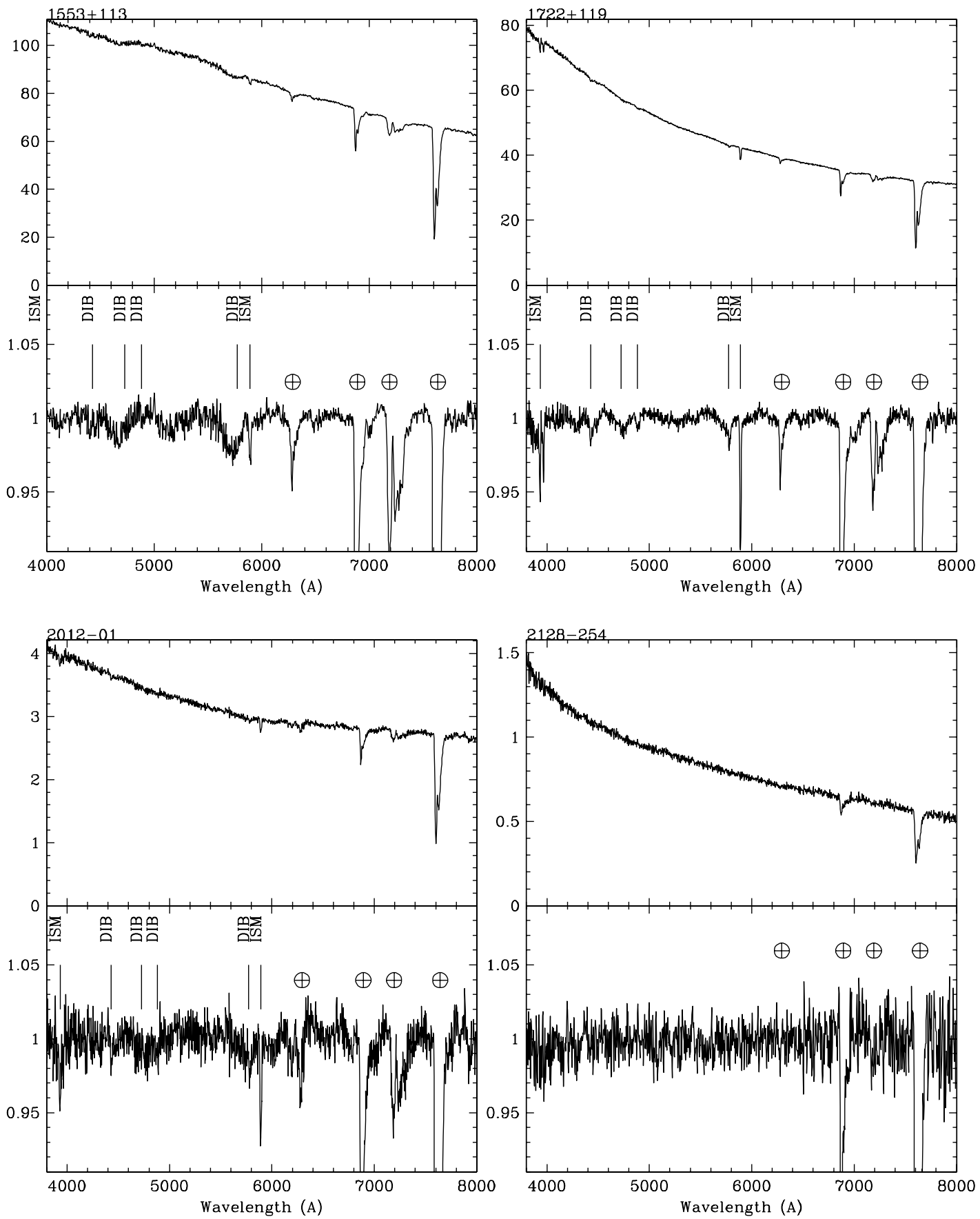

FIG. 2.-Continued

while six sources are reclassified. Depending on the observed spectral properties the objects can be assembled into three groups.

\subsubsection{Confirmed BLLs with Measured $z$}

Twelve objects belonging to this group were reported in Paper I. Six more are presented here (Table 1). Three have redshifts derived from emission lines $(0723-008, z=0.128 ; 2131-021, z=$ 1.284; and 2223 $-114, z=0.997$ ) and three from absorption lines $(1212+078, z=0.137 ; 1248-296, z=0.382$; and 2214-313, $z=0.460$ ). Details on each source are given in $\S 5$.

\subsubsection{Misclassified Objects and Objects of Uncertain Nature}

Despite their classification as BLLs in one or more input catalogs, six sources have spectra incompatible with this iden- tification. Five of them were reclassified as either quasars $(0420+022$ and $1320+084)$ or stars $(1210+121,1222+102$, and 1319+019), while object $0841+129$ remains of uncertain nature.

\subsubsection{Lineless BLLS}

In spite of the high $\mathrm{S} / \mathrm{N}, 18$ objects exhibit spectra lacking any intrinsic feature. In several spectra we clearly see absorption features from the ISM of our Galaxy. In particular, we are able to detect $\mathrm{Ca}$ II $\lambda \lambda 3934,3968, \mathrm{Na}$ I $\lambda 5892$ atomic lines, and a number of DIBs $(4428,4726,4882$, and $5772 \AA$ ) generated by complex molecules in the ISM (e.g., Galazutdinov et al. 2000 and references therein). In Figure 4 we report the average spectrum of the interstellar absorptions. In three cases absorption lines from intervening gas are detected, leading to lower limits on the 

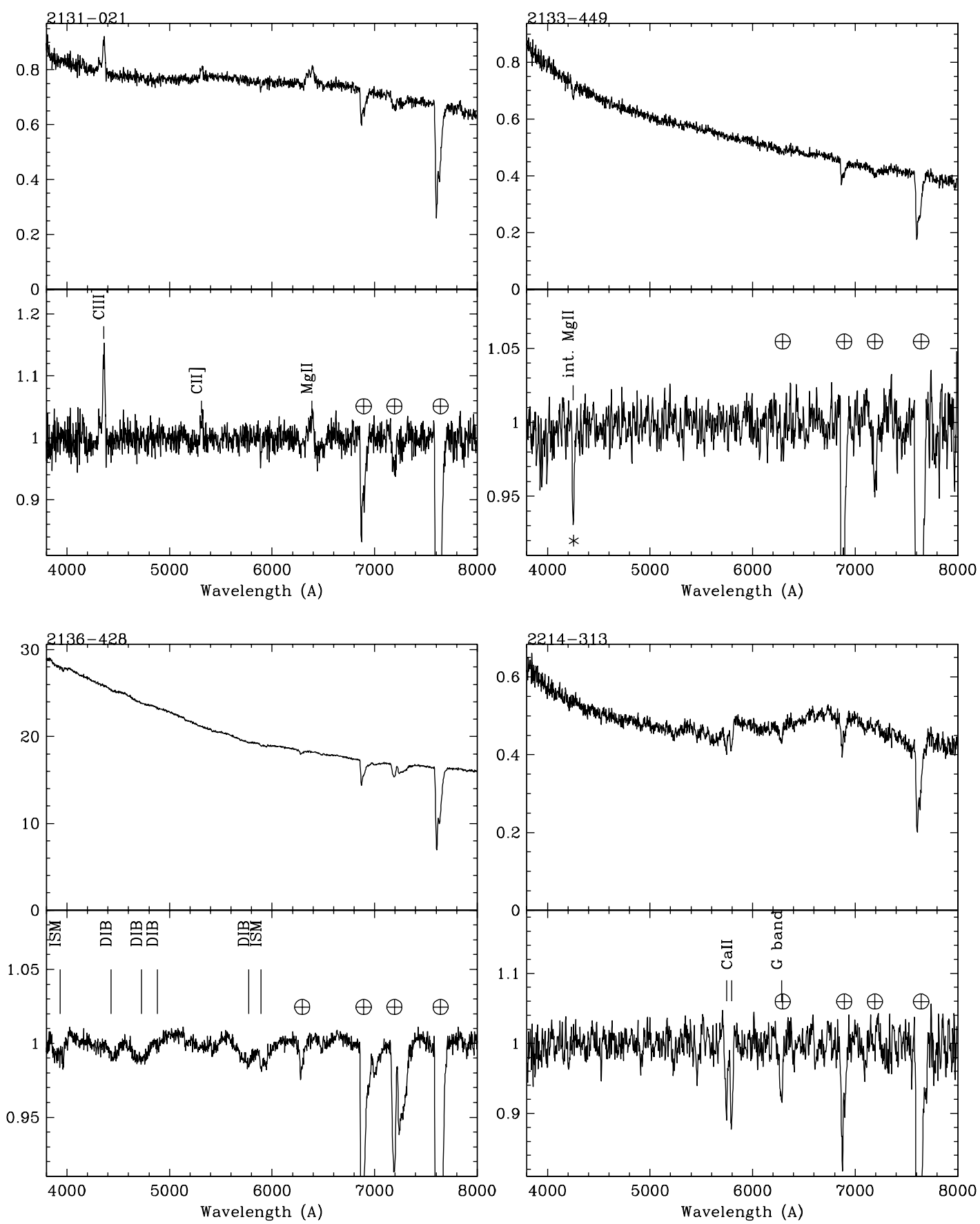

Fig. 2.-Continued

redshift of the objects $(0841+129, z>2.48 ; 2133-449, z>$ $0.52 ; 2233-148, z>0.49)$.

For these 18 sources we have estimated a redshift lower limit based on the $\mathrm{EW}_{\text {min }}$ of their spectra and the apparent magnitudes of the nuclei. We report these in Table 1. The procedure to obtain these limits is described in $\S$ 4.2.4.

\subsubsection{Redshift Lower Limits Procedure}

In this section we describe the procedure to obtain redshift lower limits for BLLs with lineless spectra (see Table 1) from the $\mathrm{EW}_{\text {min }}$ of the spectrum and the observed magnitude of the object. Under the assumption that the host galaxy luminosity is confined in a narrow range (Sbarufatti et al. 2005a) it is in fact possible to constrain the position of the source on the nucleus-tohost flux ratio $(\rho)$ versus redshift plane.
We assume that the observed spectrum of a BL Lac object is given by the contribution of two components: (1) a nonthermal emission from the nucleus that can be described by a power law $\left[F(\lambda)=C \lambda^{-\alpha}\right.$, where $C$ is the normalization constant $]$ and (2) a thermal component due to the host galaxy. Depending on the relative contribution of the two components, the optical spectrum is dominated by the nonthermal (featureless) emission or by the spectral signature of the host galaxy. The observed equivalent width $\left(\mathrm{EW}_{\mathrm{obs}}\right)$ of a given spectral absorption line is diluted depending on the ratio of the two components. Detection of this spectral feature requires a spectrum with a sufficiently high $\mathrm{S} / \mathrm{N}$. This is illustrated in Figure 5, in which a simulated spectrum $(\rho=5, z=0.5)$ is reproduced with two different $\mathrm{S} / \mathrm{N}$ ratios. The $\mathrm{S} / \mathrm{N}=300$ spectrum grants a secure detection of the Ca II features, while with $\mathrm{S} / \mathrm{N}=30$, the lines are undetected. 

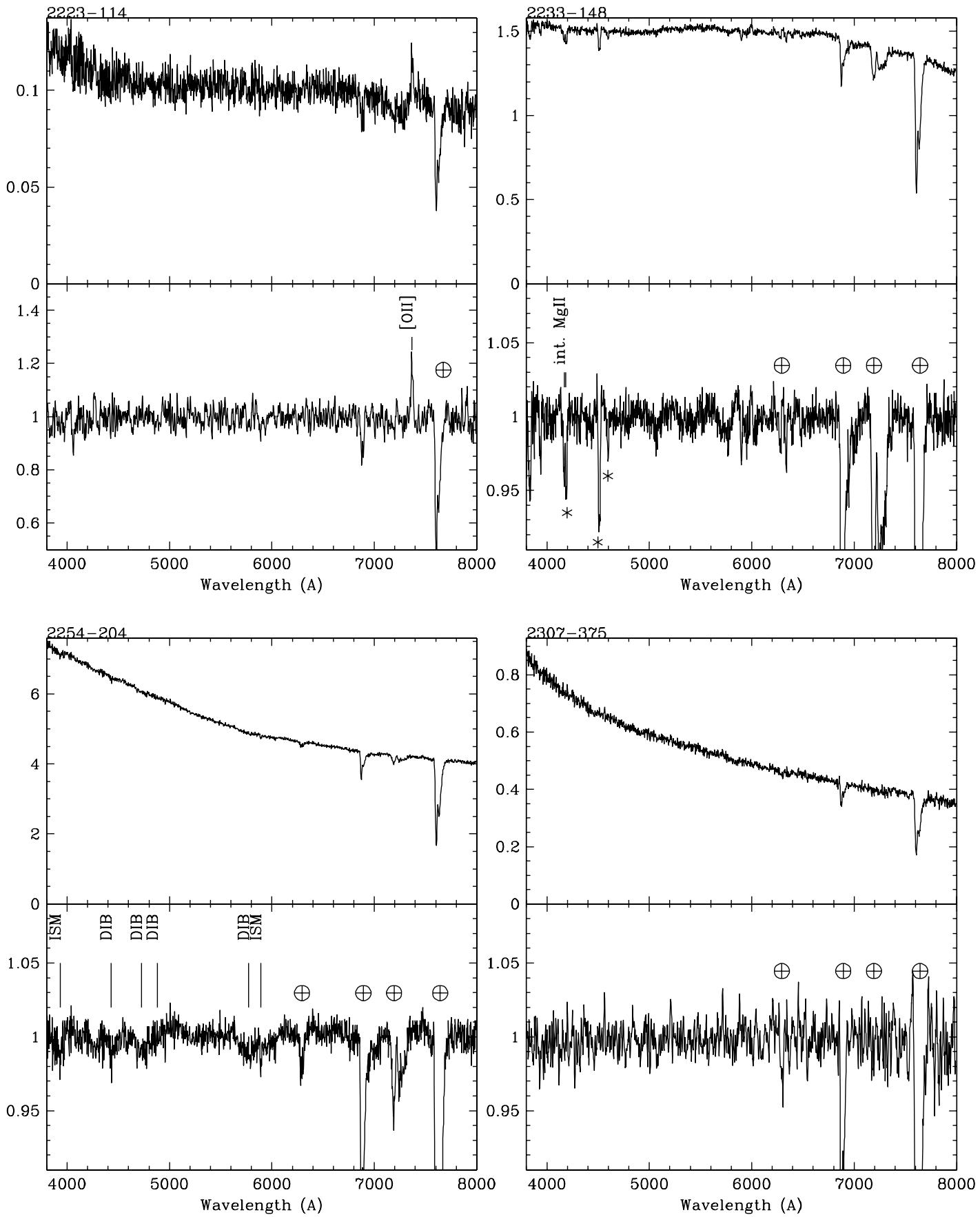

FIG. 2.-Continued

In order to estimate the redshift of an object from the $\mathrm{EW}_{\min }$, we need to know the relation between $\mathrm{EW}_{\mathrm{obs}}$ and the nucleus-tohost flux ratio $\rho$. For a spectral absorption line of intrinsic equivalent width $\mathrm{EW}_{0}$, the observed equivalent width is given by the relation (see also Sbarufatti 2005)

$$
\mathrm{EW}_{\mathrm{obs}}=\frac{(1+z) \mathrm{EW}_{0}}{1+\rho A(z)} .
$$

The nucleus-to-host ratio $\rho$ can be represented by

$$
\rho(\lambda)=\frac{F(\lambda)}{G(\lambda)},
$$

where $G(\lambda)$ is the giant elliptical galaxy spectral template by Kinney et al. (1996; see also $\S 4.1$ ), and $A(z)$ is a correction term that takes into account the loss of light inside the observed aperture. In this work the aperture is a $2^{\prime \prime} \times 6^{\prime \prime}$ slit that captures $\gtrsim 90 \%$ of the nuclear light but not the whole surrounding galaxy, which is more extended than the aperture (in particular for low- $z$ targets). In order to estimate this effect we evaluated the amount of light lost from the galaxy through the aperture in use from simulated images of BLLs (point source plus the host galaxy). The main parameters involved are the shape and the size of the host galaxy. According to the most extensive imaging studies of BLLs (Falomo 1996; Wurtz et al. 1996; Falomo \& Kotilainen 1999; Heidt et al. 1999; Nilsson et al. 2003; Urry et al. 2000), we assumed that the host is a giant elliptical galaxy of effective 

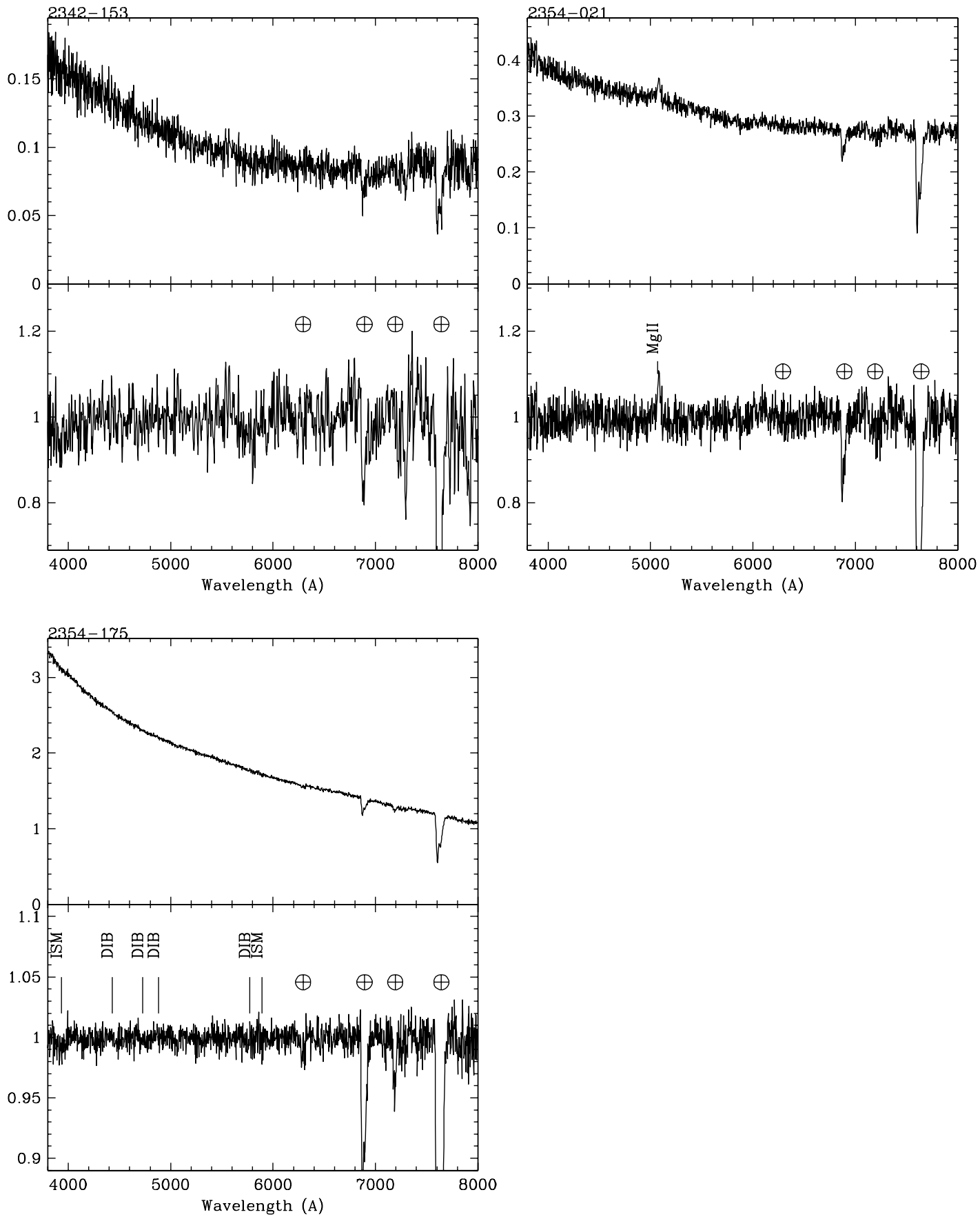

FIG. 2.-Continued

radius $R_{e}=10 \mathrm{kpc}$. The fraction of starlight lost then depends on the redshift of the object and is particularly significant at $z<0.2$, producing the bending of the curves in Figure 6 .

Since we want to refer the observed equivalent width to the nucleus-to-host ratio $\rho_{0}=\rho\left(\lambda_{0}\right)$ at a fixed wavelength $\lambda_{0}$, equation (1) can be rewritten as

$$
\mathrm{EW}_{\mathrm{obs}}=\frac{(1+z) \mathrm{EW}_{0}}{1+\rho_{0} \Delta(\lambda) A(z)}
$$

where $\Delta(\lambda)$ is the nucleus-to-host ratio normalized to that at $\lambda_{0}$ $\left[\Delta(\lambda)=\rho(\lambda) / \rho\left(\lambda_{0}\right) ;\right.$ see Fig. 7$]$.
On the other hand, the quantity $\rho_{0}$ depends also on the observed magnitudes of the object, since

$$
\log \left(\rho_{0}\right)=-0.4\left[M_{n}(z)-M_{h}(z)\right],
$$

where $M_{n}$ is the nucleus absolute magnitude and $M_{h}$ is the host absolute magnitude, and

$$
M_{n}(z)=m_{n}+5-5 \log d_{L}(z)-k_{n}(z)
$$

where $m_{n}$ is the nucleus apparent magnitude, $d_{L}(z)$ is the luminosity distance, and $k_{n}(z)$ is the nucleus $k$-correction, computed 


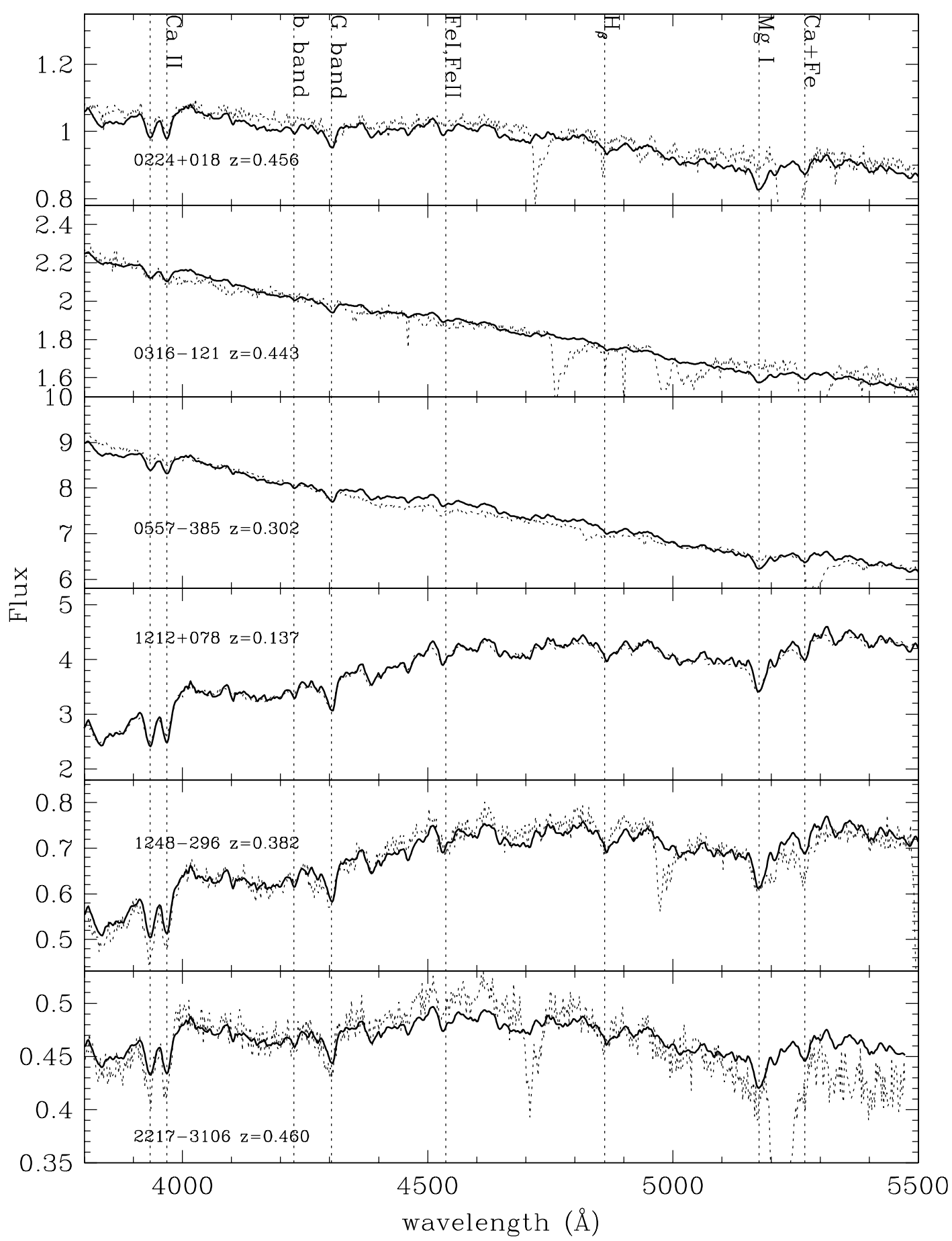

Fig. 3.-Spectral decomposition for objects with detected host galaxy spectral features in the object's rest frame. Solid lines show the fitted spectra, and dotted lines the observed ones. Objects 0224+018, 0316-121, and 0557-385 were discussed in Paper I.

following Wisotzki (2000). The absolute magnitude of the host is

$$
M_{h}(z)=M_{h}^{*}-E(z),
$$

where $M_{h}^{*}=-22.9$ is the average $R$-band magnitude of BL Lac hosts at $z=0$ and $E(z)$ is the evolution correction, as given by Bressan et al. (1998).

An example of the procedure described above is given in Figure 8, in which the relationships between $\log \left(\rho_{0}\right)$ and the redshift for a given value of $\mathrm{EW}_{\min }$ and $m_{n}$ are shown. The inter- section of the two curves yields a lower limit to the redshift of the target. When it goes beyond the observed spectral range, we set the redshift limit to the value corresponding to the considered feature reaching the upper limit of the observed wavelength range $(z \sim 1$ in the case of the Ca II 23934 line). The uncertainty of this procedure depends mainly on the spread of the distribution of the host galaxy luminosity. This issue is discussed in Urry et al. (2000) and in Sbarufatti et al. (2005a), in which it is shown that the 64 BLLs hosts of known redshift resolved with the Hubble Space Telescope (HST) are well represented by an elliptical galaxy of $M_{R}=-22.9$, with $68 \%$ of them in the interval -23.4 to -22.4 . 
TABLE 2

Parameters of BL Lac Spectral Decomposition

\begin{tabular}{ccccccc}
\hline \hline $\begin{array}{c}\text { Object } \\
(1)\end{array}$ & $\begin{array}{c}z \\
(2)\end{array}$ & $\begin{array}{c}\alpha \\
(3)\end{array}$ & $\begin{array}{c}m_{R}^{\text {host }} \\
(4)\end{array}$ & $\begin{array}{c}M_{R}^{\text {host }} \\
(5)\end{array}$ & $\begin{array}{c}\rho_{0} \\
(6)\end{array}$ & $\begin{array}{c}M_{\text {phot }}^{\text {host }} \\
(7)\end{array}$ \\
\hline $0224+018^{\mathrm{a}} \ldots \ldots \ldots \ldots \ldots \ldots \ldots$ & 0.456 & 1.50 & 19.9 & -23.2 & 2.2 & -23.1 \\
$0316-121^{\mathrm{a}} \ldots \ldots \ldots \ldots \ldots \ldots \ldots$ & 0.443 & 1.44 & 20.2 & -22.8 & 6.4 & $\ldots$ \\
$0557-385^{\mathrm{a}} \ldots \ldots \ldots \ldots \ldots \ldots \ldots$. & 0.302 & 1.61 & 18.3 & -23.4 & 5.5 & $\ldots$ \\
$1212+078 \ldots \ldots \ldots \ldots \ldots \ldots \ldots .$. & 0.137 & 1.17 & 17.4 & -22.0 & 0.4 & -23.0 \\
$1248-296 \ldots \ldots \ldots \ldots \ldots \ldots \ldots$. & 0.382 & 0.92 & 19.7 & -22.7 & 0.8 & -23.7 \\
$2214-313 \ldots \ldots \ldots \ldots \ldots \ldots \ldots$. & 0.460 & 0.90 & 20.8 & -22.3 & 2.1 & $\cdots$ \\
\hline
\end{tabular}

Notes.-Col. (1): Object name. Col. (2): Redshift. Col. (3): Fitted spectral index of the continuum, $\alpha$, defined by $F_{\lambda} \propto \lambda^{-\alpha}$. Col. (4): Fitted $R$ magnitude of the host galaxy. Col. (5): Absolute $R$ magnitude of the host galaxy, corrected for extinction and evolution but not for aperture effects. Col. (6): Rest-frame $R$-band nucleus-to-host flux ratio. Col. (7): Absolute $R$ magnitude of the host galaxy from photometry.

a Spectrum published in Paper I

This procedure can be used for any absorption line belonging to the host galaxy and for which an estimate of the undiluted equivalent width is available. In this work we considered the Ca II absorption line at $3934 \AA\left(\mathrm{EW}_{0}=16 \AA\right)$, we assumed a power-law spectral index $\alpha=0.7$ (Falomo et al. 1993), and we referred to the effective wavelength of the $R$ band $\left(\lambda_{0}=6750 \AA\right)$ to compute $\rho_{0}$ (which implies $\Delta=4.3$ ).

In order to test this procedure, we considered eight BLLs for which the $\mathrm{Ca}$ II line of the host galaxy had been measured. Five of these objects derive from the observations discussed here and in Paper I, and three others are from observations obtained at the ESO $3.6 \mathrm{~m}$ (Carangelo et al. 2003; Sbarufatti 2005). These spectra are reported in Figure 9, and the relevant parameters are given in Table 4 . The comparison between the redshifts estimated by our procedure and the spectroscopic ones indicates a reasonably good agreement (see Fig. 10).

\section{NOTES ON INDIVIDUAL OBJECTS}

0047+023: This compact and flat-spectrum radio source was classified as a BL Lac by Hewitt \& Burbidge (1993) on the basis of UV color and featureless spectra. Further featureless optical spectra were obtained by Allington-Smith et al. (1991); VéronCetty \& Véron (1993b) confirmed the BL Lac. Even in our $\mathrm{S} / \mathrm{N} \sim 80$ spectrum no spectral features were found. Based on the minimum detectable equivalent width the source is most likely at $z>0.82$.

0048-097: Previous optical observations of this well-known BL Lac object belonging to the 1 Jy sample reported a featureless spectrum (Stickel et al. 1991; Falomo et al. 1994). Rector \& Stocke (2001), however, suggested the presence of an emission line at $6092 \AA$ (possibly identified with [O II] $\lambda 3727$ at $z=0.634$ or [O II] $\lambda 5007$ at $z=0.216$ ). Falomo (1996) proposed $z>0.5$, based on the nondetection of the host galaxy in the optical images of the source. Our $\mathrm{S} / \mathrm{N}=250$ optical spectrum does not confirm the presence of the emission line at $6092 \AA$, and apart from some telluric lines and a number of Galactic absorptions, it is found to be featureless. From our $\mathrm{EW}_{\text {min }}$ estimate, we infer that this source is at $z>0.3$.

0420+022: Fricke et al. (1983) classified this source as a BL Lac candidate on the basis of a featureless (although noisy) optical spectrum. Ellison et al. (2001), through an unpublished optical spectrum, propose a redshift $z=2.277$ and classify the source as a radio-loud QSO. In our optical spectrum we are able to clearly detect emission lines $\operatorname{Ly} \alpha \lambda 1419, \mathrm{O}$ vI] $\lambda 1034, \mathrm{C}$ IV $\lambda 1549$, and C III] $\lambda 1909$, at $z=2.278$. A recent spectrum ob-

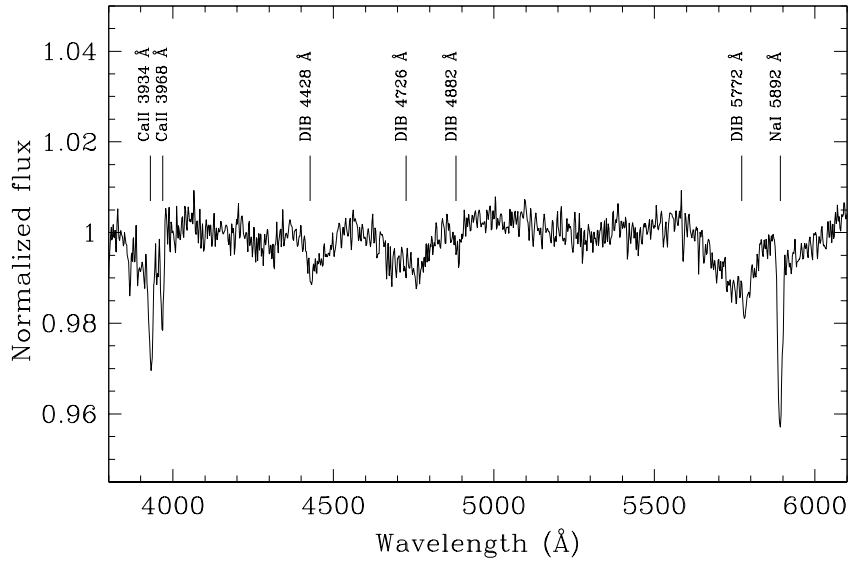

FIG. 4.-Combined spectrum of the ISM features of lineless BLLs. Ca II $\lambda \lambda 3934,3968$ and $\mathrm{Na}$ I $\lambda 5892$ atomic lines and DIBs at 4428, 4726, 4882, and $5772 \AA$ are indicated.

tained by Hook et al. (2003) also confirms our findings and the classification of the object as a QSO.

0422+004: This object is a well-known radio-selected BL Lac, included in the Hewitt \& Burbidge (1993) catalog. Falomo (1996) detected the host galaxy with ground-based imaging, proposing $z \sim 0.2-0.3$. The optical spectrum taken by Falomo et al. (1994) is featureless. Our spectrum $(\mathrm{S} / \mathrm{N}=230)$ does not show evidence for intrinsic spectral features from the host, suggesting a very high $\mathrm{N} / \mathrm{H}$ ratio. Interstellar absorption from $\mathrm{Na}$ I $\lambda 5892$ and DIBs at 5772 and $4726 \AA$ is well detected. Based on $\mathrm{EW}_{\text {min }}$, we estimate $z>0.31$.

0627-199: Hook et al. (2003) obtained a lineless spectrum for this radio-selected BL Lac object. Our VLT spectrum, of moderate $\mathrm{S} / \mathrm{N}(50)$, shows no spectral features. From $\mathrm{EW}_{\min }$ we set $z>0.63$.

0723-008: Wills \& Wills (1976) classified this source as a narrow emission line radio galaxy based on an optical spectrum, giving $z=0.127$. Rusk \& Seaquist (1985) reported an optical polarization of $1.5 \%$, classifying the source as a low-polarization QSO. Véron-Cetty \& Véron (2001) reported this source as a BL Lac object. Henriksen et al. (1984) gave broadband indices $\alpha_{\text {ro }}=0.7$ and $\alpha_{\text {ox }}=1.0$, which are compatible with a BL Lac or a FSRQ classification. Our optical spectrum is clearly dominated by nonthermal emission with spectral index $\alpha=0$.7. Superposed on this, strong narrow emission lines and absorption lines from the underlying host galaxy at $z=0.127$ are detected, confirming the redshift. From the values of the spectral indices and the measured EW for the spectral lines, we suggest that this object is of intermediate nature between a BL Lac and a quasar.

0841+129: This source, first identified by C. Hazard (see Jaunsen et al. 1995 and references therein), is a damped Ly $\alpha$ absorption (DLA) QSO at $z>2.48$ as derived from the two very strong DLAs at $\sim 4100$ and $\sim 4225 \AA$ (see, e.g., Pettini et al. 1997; Prochaska et al. 2001; Warren et al. 2001 and references therein). The classification as a BL Lac object was motivated by the absence of prominent emission lines (Hewitt \& Burbidge 1993).

Our spectrum, in addition to several absorption lines, exhibits three possible broad emission structures at $\sim 4310, \sim 4850$, and $\sim 5370 \AA$. These could be interpreted as N v $\lambda 1240$, Si Iv $\lambda 1397$, and $\mathrm{C}$ IV $\lambda 1549$ at $z \sim 2.47$. This is consistent with $z \sim 2.5$, deduced from the observed position of the onset of the absorption of the Ly $\alpha$ forest (Warren et al. 2001). An alternative explanation, however, is that these structures are pseudoemissions 
TABLE 3

Measurements of Spectral Lines

\begin{tabular}{|c|c|c|c|c|c|c|c|c|}
\hline $\begin{array}{c}\text { Object Name } \\
\text { (1) }\end{array}$ & $\begin{array}{c}\text { Object Class } \\
\text { (2) }\end{array}$ & $\begin{array}{l}z_{\text {avg }} \\
(3)\end{array}$ & $\begin{array}{l}\text { Line ID } \\
\text { (4) }\end{array}$ & $\begin{array}{c}\lambda \\
(5)\end{array}$ & $\begin{array}{c}z \\
(6)\end{array}$ & $\begin{array}{l}\text { Type } \\
(7)\end{array}$ & $\begin{array}{l}\text { FWHM } \\
\text { (8) }\end{array}$ & $\begin{array}{l}\text { EW } \\
(9)\end{array}$ \\
\hline \multirow[t]{7}{*}{$0420+022$} & QSO & 2.278 & & & & & & \\
\hline & & & $\operatorname{Ly} \alpha$ & 4020 & 2.278 & $\mathrm{e}$ & 9400 & -73.0 \\
\hline & & & Si II & 4285 & 2.279 & $\mathrm{e}$ & 3900 & -4.0 \\
\hline & & & $\mathrm{C}_{\text {II }}$ & 4381 & 2.281 & $\mathrm{e}$ & 5500 & -3.0 \\
\hline & & & Si IV & 4587 & 2.283 & $\mathrm{e}$ & 6300 & -24.0 \\
\hline & & & $\mathrm{C}_{\text {IV }}$ & 5077 & 2.278 & $\mathrm{e}$ & 4900 & -50.0 \\
\hline & & & $\mathrm{C}$ III] & 6250 & 2.274 & $\mathrm{e}$ & 4500 & -45.0 \\
\hline \multirow[t]{16}{*}{$0723-008$} & QSO/BLL & 0.127 & & & & & & \\
\hline & & & {$[\mathrm{Ne} v]$} & 3858 & 0.126 & $\mathrm{e}$ & 1100 & -0.4 \\
\hline & & & {$[\mathrm{O}$ II $]$} & 4200 & 0.127 & $\mathrm{e}$ & 1200 & -2.1 \\
\hline & & & [Ne III] & 4359 & 0.127 & $\mathrm{e}$ & 1300 & -0.7 \\
\hline & & & $\mathrm{Ca}$ II & 4433 & 0.127 & $\mathrm{~g}$ & $\ldots$ & 0.6 \\
\hline & & & $b$ band & 4477 & 0.127 & $\mathrm{~g}$ & $\ldots$ & 0.1 \\
\hline & & & $G$ band & 4847 & 0.127 & $\mathrm{~g}$ & $\ldots$ & 0.1 \\
\hline & & & $\mathrm{H} \gamma$ & 4897 & 0.128 & $\mathrm{e}$ & 3700 & -1.0 \\
\hline & & & $\mathrm{H} \beta$ & 5477 & 0.127 & $\mathrm{e}$ & 1100 & -1.0 \\
\hline & & & [O III] & 5587 & 0.127 & $\mathrm{e}$ & 900 & -1.9 \\
\hline & & & {$[\mathrm{O} \quad \mathrm{III}]$} & 5642 & 0.127 & $\mathrm{e}$ & 900 & -5.5 \\
\hline & & & $\mathrm{Mg}_{\mathrm{I}}$ & 5830 & 0.127 & $\mathrm{~g}$ & $\ldots$ & 0.4 \\
\hline & & & $\mathrm{Na} I$ & 6645 & 0.127 & $\mathrm{~g}$ & $\ldots$ & 0.1 \\
\hline & & & {$[\mathrm{O}$ II] } & 7098 & 0.127 & $\mathrm{e}$ & 1000 & -1.2 \\
\hline & & & $\mathrm{H} \alpha$ & 7402 & 0.128 & $\mathrm{e}$ & 1900 & -8.7 \\
\hline & & & $\mathrm{S}$ II & 7561 & 0.126 & e & 600 & -1.1 \\
\hline \multirow[t]{8}{*}{ 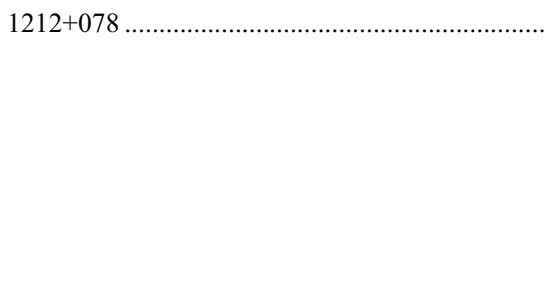 } & BLL & 0.137 & & & & & & \\
\hline & & & $\mathrm{Ca}$ II & 4473 & 0.137 & $\mathrm{~g}$ & $\ldots$ & 5.7 \\
\hline & & & $\mathrm{Ca}_{\mathrm{II}}$ & 4510 & 0.137 & $\mathrm{~g}$ & $\ldots$ & 4.8 \\
\hline & & & $G$ band & 4890 & 0.136 & $\mathrm{~g}$ & $\cdots$ & 5.3 \\
\hline & & & $\mathrm{H} \beta$ & 5529 & 0.137 & $\mathrm{~g}$ & $\ldots$ & 3.5 \\
\hline & & & $\mathrm{Mg}$ I & 5883 & 0.137 & $\mathrm{~g}$ & $\ldots$ & 16.2 \\
\hline & & & $\mathrm{Na} I$ & 6696 & 0.137 & $\mathrm{~g}$ & $\ldots$ & 3.9 \\
\hline & & & $\mathrm{H} \alpha$ & 7481 & 0.139 & $\mathrm{e}$ & 700 & -2.0 \\
\hline \multirow[t]{5}{*}{ 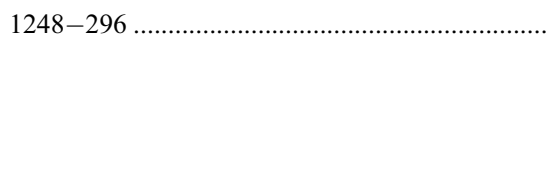 } & BLL & 0.382 & & & & & & \\
\hline & & & $\mathrm{Ca}$ II & 5436 & 0.382 & $\mathrm{~g}$ & 2100 & 6.1 \\
\hline & & & $\mathrm{Ca}_{\text {II }}$ & 5482 & $\ldots$ & $\mathrm{g}$ & 1700 & 4.2 \\
\hline & & & $G$ band & 5942 & $\ldots$ & $\mathrm{g}$ & 2700 & 5.6 \\
\hline & & & $\mathrm{H}_{\beta}$ & 6719 & 0.382 & $\mathrm{~g}$ & 1600 & 1.5 \\
\hline \multirow[t]{11}{*}{ 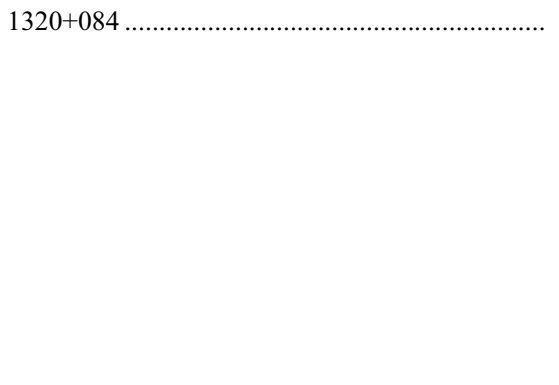 } & QSO & 1.500 & & & & & & \\
\hline & & & C IV & 3873 & 1.500 & $\mathrm{e}$ & 2200 & 1.9 \\
\hline & & & He II & 4095 & 1.500 & $\mathrm{e}$ & 1900 & -9.0 \\
\hline & & & $?$ & 4234 & $\ldots$ & $\mathrm{a}$ & $\ldots$ & -80.2 \\
\hline & & & $\mathrm{N}$ III] & 4376 & 1.500 & $\mathrm{e}$ & 2300 & -5.8 \\
\hline & & & $\mathrm{C}$ III] & 4770 & 1.500 & e & 2200 & -40.9 \\
\hline & & & $?$ & 6071 & $\ldots$ & $\mathrm{a}$ & $\ldots$ & 0.6 \\
\hline & & & $?$ & 6130 & $\ldots$ & $\mathrm{a}$ & $\ldots$ & 1.4 \\
\hline & & & $\mathrm{Mg}$ II & 6563 & 1.347 & $\mathrm{a}$ & $\ldots$ & 1.8 \\
\hline & & & $\mathrm{Mg}_{\mathrm{II}}$ & 6578 & 1.347 & $\mathrm{a}$ & $\ldots$ & 1.6 \\
\hline & & & $\mathrm{Mg}$ II & 7001 & 1.502 & $\mathrm{e}$ & 6700 & -60.0 \\
\hline \multirow[t]{4}{*}{ 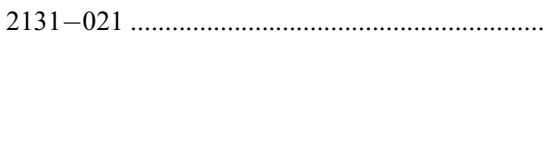 } & BLL & 1.283 & & & & & & \\
\hline & & & $\mathrm{C}$ III] & 4357 & 1.283 & $\mathrm{e}$ & 2000 & -4.4 \\
\hline & & & $\mathrm{C}$ II] & 5312 & 1.284 & $\mathrm{e}$ & 700 & -1.4 \\
\hline & & & $\mathrm{Mg}_{\text {II }}$ & 6383 & 1.281 & $\mathrm{e}$ & 3000 & -3.8 \\
\hline \multirow[t]{2}{*}{ 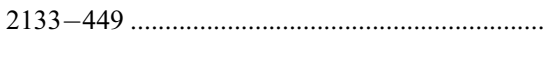 } & BLL & $>0.52$ & & & & & & \\
\hline & & & $\mathrm{Mg}$ II & 4250 & 0.519 & $\mathrm{a}$ & 2500 & 1.5 \\
\hline \multirow[t]{4}{*}{ 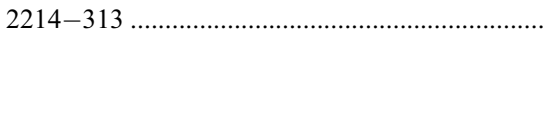 } & BLL & 0.460 & & & & & & \\
\hline & & & $\mathrm{Ca}$ II & 5746 & 0.461 & $\mathrm{~g}$ & 3000 & 3.7 \\
\hline & & & $\mathrm{Ca}$ II & 5792 & 0.460 & $\mathrm{~g}$ & 4300 & 3.3 \\
\hline & & & $G$ band & 6280 & 0.459 & $\mathrm{~g}$ & 3200 & 2.6 \\
\hline \multirow[t]{2}{*}{$2223-114 \ldots$} & BLL & 0.977 & & & & & & \\
\hline & & & {$[\mathrm{O}$ II] } & 7367 & $\ldots$ & $\mathrm{e}$ & 1200 & -5.0 \\
\hline \multirow[t]{5}{*}{$2233-148$} & BLL & $>0.49$ & & & & & & \\
\hline & & & $\mathrm{Mg}$ II & 4165 & 0.490 & $\mathrm{a}$ & $\ldots$ & 0.7 \\
\hline & & & $\mathrm{Mg}$ II & 4183 & 0.493 & $\mathrm{a}$ & $\ldots$ & 0.9 \\
\hline & & & $?$ & 4514 & $\ldots$ & $\mathrm{a}$ & $\ldots$ & 1.7 \\
\hline & & & $?$ & 4598 & $\ldots$ & $\mathrm{a}$ & $\ldots$ & 0.5 \\
\hline
\end{tabular}

Notes: Col. (1): Object name. Col. (2): Object class. Col. (3): Average redshift. Col. (4): Line identification. Col. (5): Observed wavelength of line center (Å).

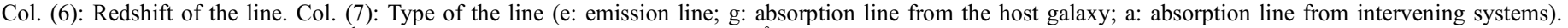
Col. (8): FWHM of the line $\left(\mathrm{km} \mathrm{s}^{-1}\right)$. Col. (9): Equivalent width of the line $(\AA)$. 

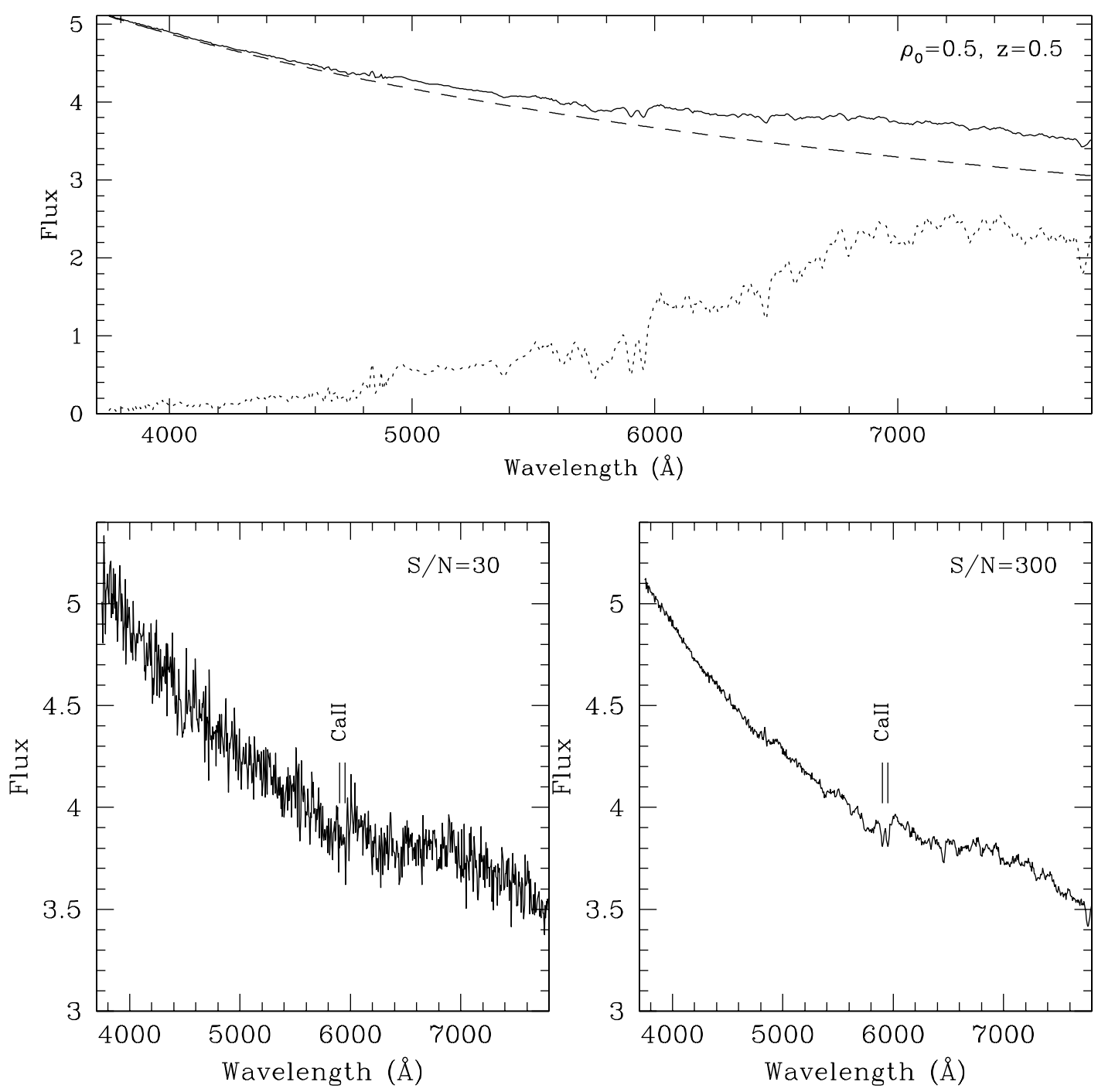

FIG. 5.-Top: Simulated BL Lac spectrum at $z=0.5$ (solid line), obtained as the composition of a nonthermal power law (dashed line) and an elliptical galaxy spectrum (magnified 5 times, dotted line), with nucleus-to-host ratio of 5. Bottom: Simulated spectrum, if observed with $\mathrm{S} / \mathrm{N}=30$ (left) or $\mathrm{S} / \mathrm{N}=300$ (right).

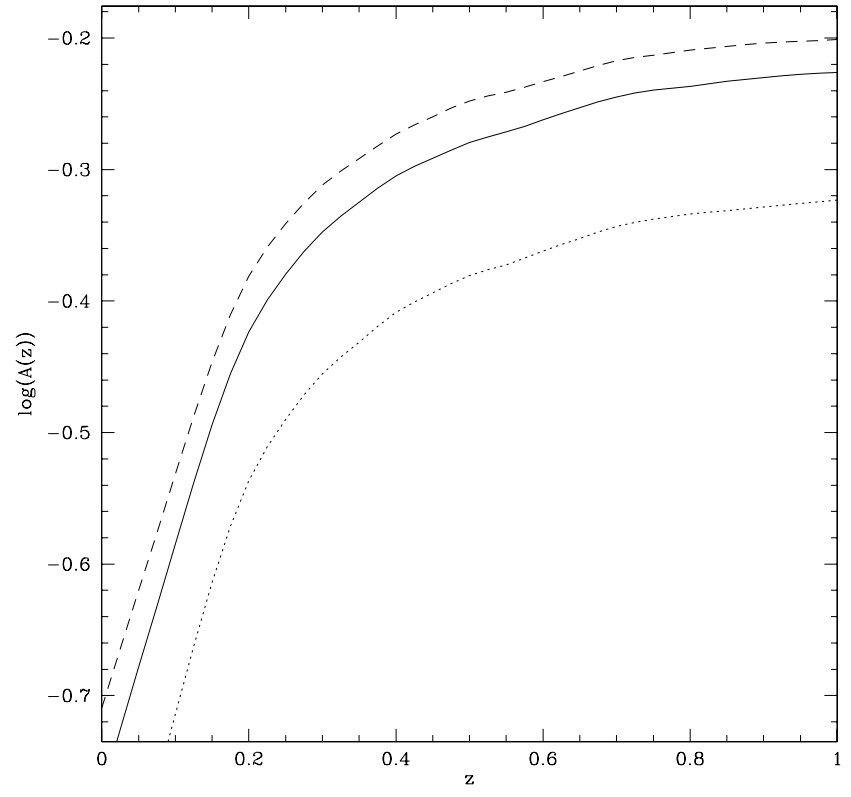

FIG. 6.-Aperture effect correction $A(z)$ as a function of the redshift for aperture sizes $2^{\prime \prime} \times 12^{\prime \prime}$ (dashed line), $2^{\prime \prime} \times 6^{\prime \prime}$ (solid line), and $2^{\prime \prime} \times 3^{\prime \prime}$ (dotted line). Seeing is assumed to be $\sim 1^{\prime \prime}$. resulting from the depression of the continuum caused by the envelope of many unresolved narrow absorption features. Higher resolution spectra of the object in the spectral range 4200-5800 $\AA$ are needed to distinguish between the two possibilities.

1210+121: Hazard \& Murdoch (1977) proposed that this object was the optical counterpart of a radio source in the Molongo Catalogue (Sutton et al. 1974); the separation was, however, $16^{\prime \prime}$. Zotov \& Tapia (1979) reported large optical variability and polarization, apparently reinforcing the identification. Baldwin et al. (1973) found a featureless optical spectrum. Our VLT spectrum clearly shows that the source is a type B star in our Galaxy.

1212+078: Our VLT spectrum clearly shows the presence of a strong thermal component due to the host. We detected Ca II $\lambda \lambda 3934,3968, G$ band $\lambda 4305, \mathrm{Mg}$ I $\lambda 5175$, and $\mathrm{H} \alpha \lambda 6563$ in emission at $z=0.137$, confirming the redshift estimated by Perlman et al. (1996). The contribution of the nonthermal component is visible in the bluest part of the spectrum. The best-fit decomposition of the spectrum gives $\alpha=1.17$ for the nonthermal component and $M_{R}=-22.0$ for the host. Although this is somewhat fainter than expected for a BL Lac host galaxy, we cannot exclude the possibility that part of the light did not enter in the slit, given the low redshift and the consequent large apparent size of the host. 


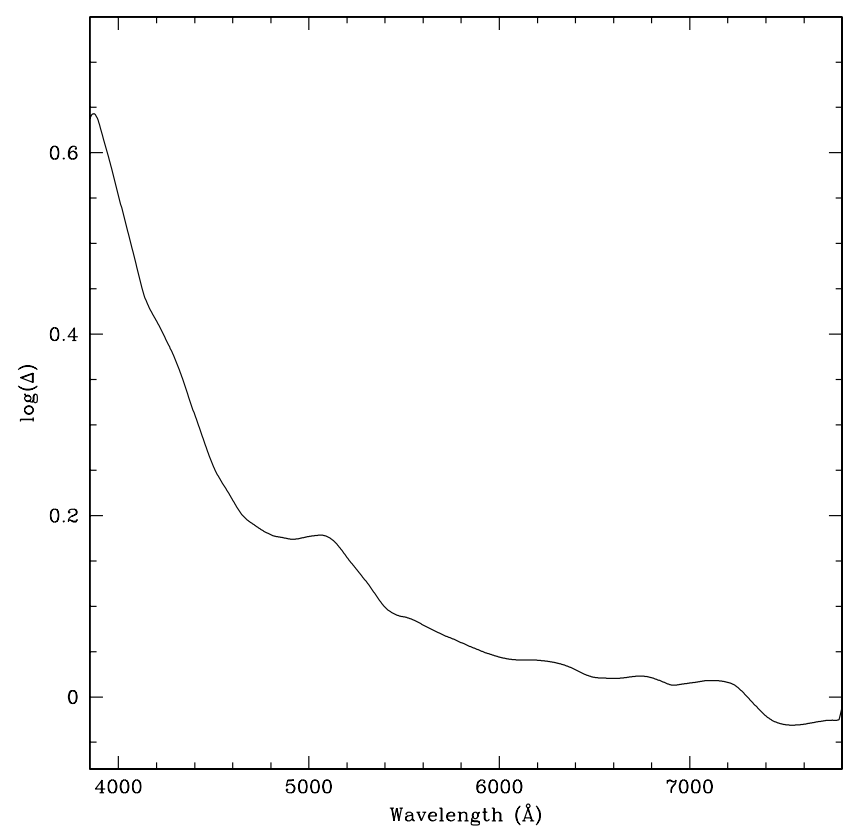

FIG. 7.-Relative nucleus-to-host ratio $\Delta$, referred to $\lambda_{0}=6750 \AA$ (effective wavelength for $R$-band magnitude), as a function of the wavelength. The assumed spectral index for the nuclear component is $\alpha=0.7$. For the Ca II $\lambda 3934$ absorption feature, $\Delta=4.3$.

1222+102: This is a blue stellar object in the direction of the Virgo-Coma Cluster. Its apparent position in the sky is very close to the center of the galaxy NGC 4380, still well inside the galaxy boundaries. The projected separation to the nucleus at the redshift of the galaxy is $\sim 10 \mathrm{kpc}$. The object is considered a candidate BL Lac in the Burbidge \& Hewitt (1987) list, selected because of its UV excess. Arp (1977) reports the observation of a featureless spectrum. Burbidge (1996) considers this object as a possible candidate of expulsion from a galactic nucleus. The sharp absorption lines detected in our spectrum clearly indicate a stellar origin. The measured colors lead to a temperature of $\sim 10,000 \mathrm{~K}$. If the object were a main-sequence or a supergiant star, the corresponding distance would put it outside the Galaxy but not at the distance of NGC 4380. We are therefore led to consider it to be a white dwarf, which would be at $100-$ $200 \mathrm{pc}$. The absence of $\mathrm{H}$ lines indicates a DQ or DXP white dwarf (Schmidt et al. 2001, 2003). Some of the lines are referable to $\mathrm{He} \mathrm{I}$ and $\mathrm{C}$ I transitions. The object clearly deserves further study; in particular, polarization measurements would be interesting.

1248-296: Perlman et al. (1996) obtained a low S/N spectrum of this source and proposed it to be a BL Lac at $z=0.487$ based on the possible detection of the host galaxy features. In our VLT spectrum $\mathrm{Ca}$ II, $\mathrm{G}$ band, and $\mathrm{H} \beta$ are clearly detected at $z=0.382$, confirming the findings of Woo et al. (2005), while in the blue part the contribution of a nonthermal component is clearly visible. The best-fit decomposition gives $\alpha=0.92$ for the nonthermal component visible below $5000 \AA$, and $M_{R}=-22.7$ for the host, in good agreement with the result from the direct detection of the host in HST imaging (Urry et al. 2000; Sbarufatti et al. 2005a).

1319+019: This object was initially selected as a BL Lac candidate on the basis of the University of Michigan objective prism survey (MacAlphine \& Williams 1981) designed to find AGNs, and it is included as a BL Lac in the Véron-Cetty \& Véron (2001) catalog. No radio counterpart for this source has been

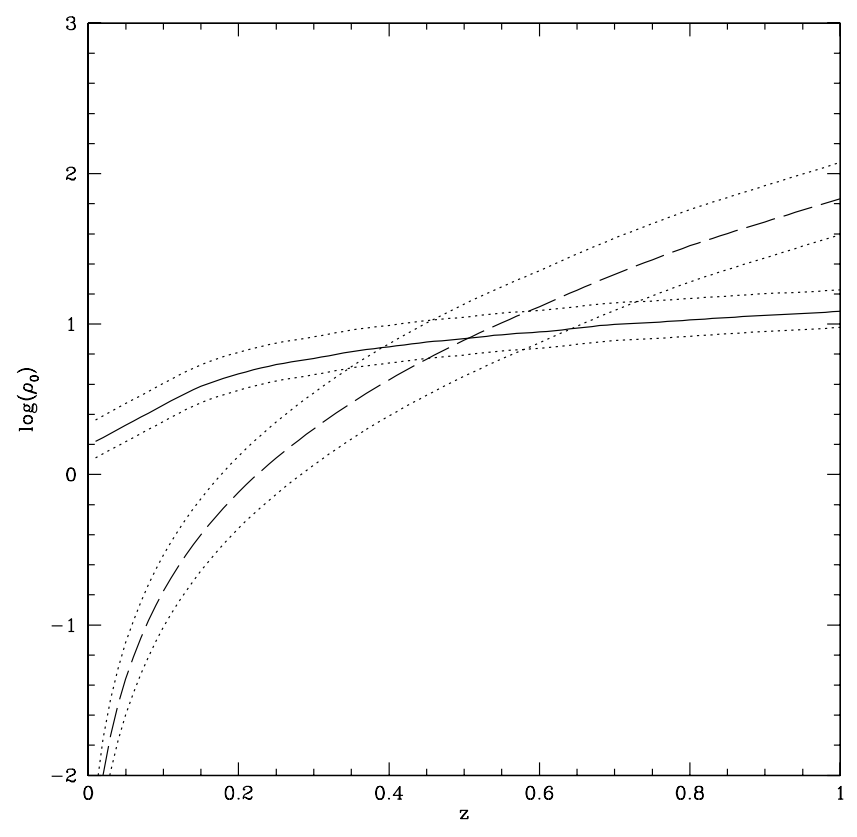

FIG. 8. - Redshift lower limit obtained from apparent magnitude and $\mathrm{EW}_{\min }$ values for 1RXS J150343.0-154107. The thick solid line represents the N/H vs. $z$ limit obtained from the $\mathrm{EW}_{\min }$ value. Dotted curves correspond to a $0.1 \AA$ uncertainty in $\mathrm{EW}_{\min }$. The dashed line gives the $\mathrm{N} / \mathrm{H}$ vs. $z$ relation for a $\mathrm{BL}$ Lac with a host galaxy with $M_{R}=-22.9$ and nuclear apparent magnitude $R=17.7$. Dotted lines correspond to the uncertainty due to the range of variation of host galaxy magnitude $(0.5 \mathrm{mag})$ and observational photometric errors $(0.1 \mathrm{mag})$. The intersection between the two solid lines gives the lower limit on the redshift. The analytic form of the curves is described by eqs. (3) and (4); further details can be found in Sbarufatti (2005).

found in the literature. Later, Thompson \& Djorgovski (1990) proposed its classification as a BL Lac, based on a low-S/N optical spectrum that was found to be featureless. In our much better quality spectrum we clearly see many absorption features that characterize the object as a Galactic star of spectral type $\sim \mathrm{A}$. Our findings are also in agreement with the spectral classification of the 2dF QSO Redshift Survey (2QZ; see Croom et al. 2004).

1320+084: This source is part of the BL Lac sample extracted from the Einstein Slew Survey, and a radio counterpart was reported by Perlman et al. (1996). Our VLT data show that the source has a QSO-like spectrum at $z=1.5$, in contrast with the featureless spectrum observed by Perlman et al. (1996). Several intervening absorption lines, in particular Mg II at $z=1.347$, were also detected.

1349-439: The spectrum of this X-ray selected BL Lac (della Ceca et al. 1990) shows a number of absorption lines from the ISM: Ca II $\lambda \lambda 3934,3968$, the $5772 \AA \mathrm{DIB}$, and Na I $\lambda 5892$. No intrinsic features were detected, and the deduced redshift lower limit is $z>0.39$. As already pointed out by Veron (1996), the value $z=0.05$ sometimes reported for this object is a consequence of confusion with the nearby Seyfert 1 galaxy Q1349-439.

1442-032: This X-ray source, the radio counterpart of which was found in the NVSS, was first classified as a BL Lac in the RBSC-NVSS sample by Bauer et al. (2000) and then confirmed by the SS. There are no published optical spectra for this source. Our optical spectrum is featureless, with the exception of the $\mathrm{Na}$ I $\lambda 5892$ absorption feature from our Galactic ISM. The $\mathrm{EW}_{\text {min }}$ value for this objects leads to $z>0.51$.

1500-154: This X-ray-selected BL Lac is part of the RSBCNVSS sample (Bauer et al. 2000) and enters the SS. No previous 


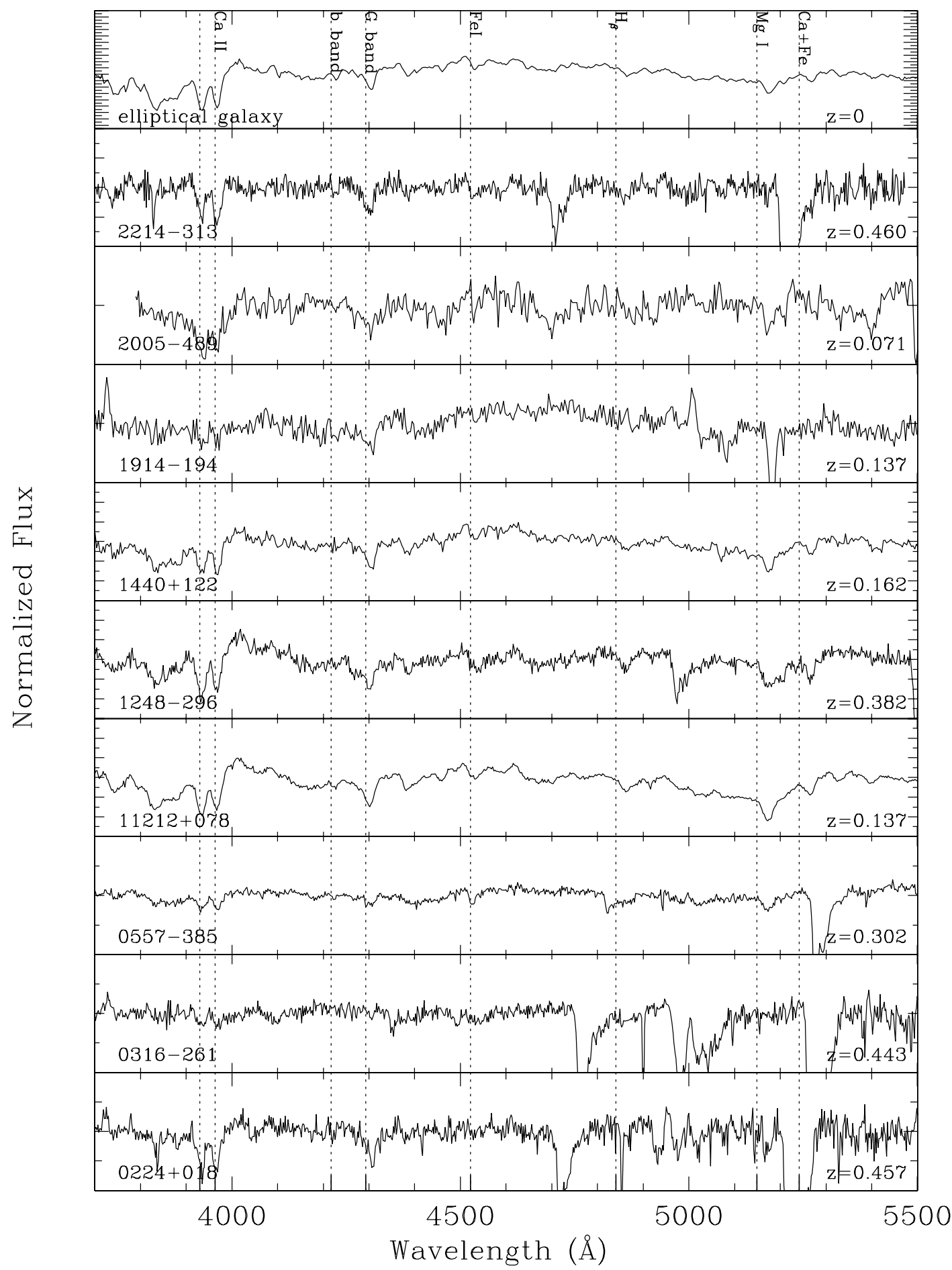

FIG. 9. - Rest-frame normalized spectra of objects used to compare redshift estimates from Ca II equivalent widths with spectroscopic values.

optical spectroscopy has been found in the literature. Our spectrum is completely featureless, leading to $z>0.38$ from the obtained $\mathrm{EW}_{\text {min }}$.

1553+113: This source is an optically selected BL Lac from the PG survey. The redshift estimate $(z=0.360)$ given in the Hewitt \& Burbidge (1993) catalog was disproved by later spectroscopy (Falomo \& Treves 1990; Falomo et al.1994). While no intrinsic features were detected in our $\mathrm{S} / \mathrm{N}=250$ VLT spectrum, a number of absorption lines due to our Galaxy's ISM were revealed: Ca II $\lambda \lambda 3934,3968, \mathrm{Na}$ I $\lambda 5892$, and DIBs at 4428, 4726,
4882, and $5772 \AA$. The $\mathrm{EW}_{\min }$ estimate for this object gives a limit of $z>0.09$.

1722+119: Griffiths et al. (1989) reported a tentative redshift $z=0.018$ for this X-ray-selected, highly polarized BL Lac. This estimate was not confirmed by more recent observations (VéronCetty \& Véron 1993b; Falomo et al. 1993, 1994). Our VLT spectrum $(\mathrm{S} / \mathrm{N}=350)$ shows only absorption features due to our Galaxy's ISM: Ca II $\lambda \lambda 3934,3968, \mathrm{Na}$ I $\lambda 5892$, and DIBs at 4428, 4726, 4882, and $5772 \AA$, with no evidence of intrinsic features. From the minimum $\mathrm{EW}_{\min }$ we derive $z>0.17$. 
TABLE 4

Comparison between Spectroscopic Redshifts and Estimates Obtained From $\mathrm{EW}_{\text {min }} \mathrm{V}_{\text {alues }}$

\begin{tabular}{ccccccc}
\hline \hline $\begin{array}{c}\text { Object Name } \\
(1)\end{array}$ & $\begin{array}{c}z \\
(2)\end{array}$ & $\begin{array}{c}m_{R}^{\text {nucleus }} \\
(3)\end{array}$ & $\begin{array}{c}\mathrm{EW}_{\mathrm{Ca} \text { II }} \\
(4)\end{array}$ & $\begin{array}{c}z_{\text {est }} \\
(5)\end{array}$ & $\begin{array}{c}\delta_{z_{\text {est }}} \\
(6)\end{array}$ & $\begin{array}{c}\text { Telescope } \\
(7)\end{array}$ \\
\hline $0224+018 \ldots \ldots$. & 0.457 & $19.0^{*}$ & 1.7 & 0.42 & 0.12 & VLT \\
$0316-261 \ldots \ldots$. & 0.443 & $18.1^{*}$ & 0.6 & 0.47 & 0.15 & VLT \\
$0557-385 \ldots \ldots$. & 0.302 & $16.9^{*}$ & 0.9 & 0.22 & 0.08 & VLT \\
$1212+078 \ldots \ldots$. & 0.137 & $18.5^{*}$ & 5.7 & 0.14 & 0.05 & VLT \\
$1248-296 \ldots \ldots$. & 0.382 & $19.8^{*}$ & 6.1 & 0.28 & 0.08 & VLT \\
$1440+122 \ldots \ldots .$. & 0.162 & 17.2 & 3.5 & 0.09 & 0.04 & ESO 3.6 m \\
$1914-194 \ldots \ldots$. & 0.137 & 15.8 & 0.5 & 0.17 & 0.09 & ESO 3.6 m \\
$2005-489 \ldots \ldots$. & 0.071 & 14.1 & 0.4 & 0.07 & 0.05 & ESO 3.6 m \\
$2214-313 \ldots \ldots$. & 0.460 & $19.9^{*}$ & 3.7 & 0.41 & 0.10 & VLT \\
\hline
\end{tabular}

Notes.-Col. (1): Object name. Col. (2): Spectroscopic $z$. Col. (3): Nucleus apparent $R$ magnitude (asterisks signify being obtained from spectral decomposition). Col. (4): Equivalent width of Ca II 23934. Col. (5): Estimated $z$. Col. (6): Error on estimated $z$. Col. (7): Telescope used for observations.

2012-017: Consistent with previous observations of this radio-selected BL Lac (White et al. 1988; Véron et al. 1990; Falomo et al. 1994), our $\mathrm{S} / \mathrm{N}=130$ VLT spectrum is also featureless. The optical spectral index is $\alpha=0.49$, in marginal agreement with $\alpha=0.33 \pm 0.12$ reported by Falomo et al. (1994). From $\mathrm{EW}_{\min }$ we derive $z>0.94$.

2128-254: The spectrum of this X-ray-selected BL Lac candidate is reported as featureless by SS. We confirm this result and set a lower limit of $z>0.86$ for the redshift.

2131-021: Rector \& Stocke (2001) and Drinkwater et al. (1997) proposed a redshift of 1.285 for this source, based on the detection of C III] $\lambda 1909, \mathrm{Mg}_{\text {II }} \lambda 2798$, and [O II] $\lambda 3727$, opposed to the $z=0.557$ suggested by Wills \& Wills (1976). While [O II] falls outside our spectral range, we confirm the presence of $\mathrm{C}$ III] and $\mathrm{Mg}$ II emission lines at $z=1.283$, also detecting the fainter $\mathrm{C}$ II] 22326 feature at the same redshift.

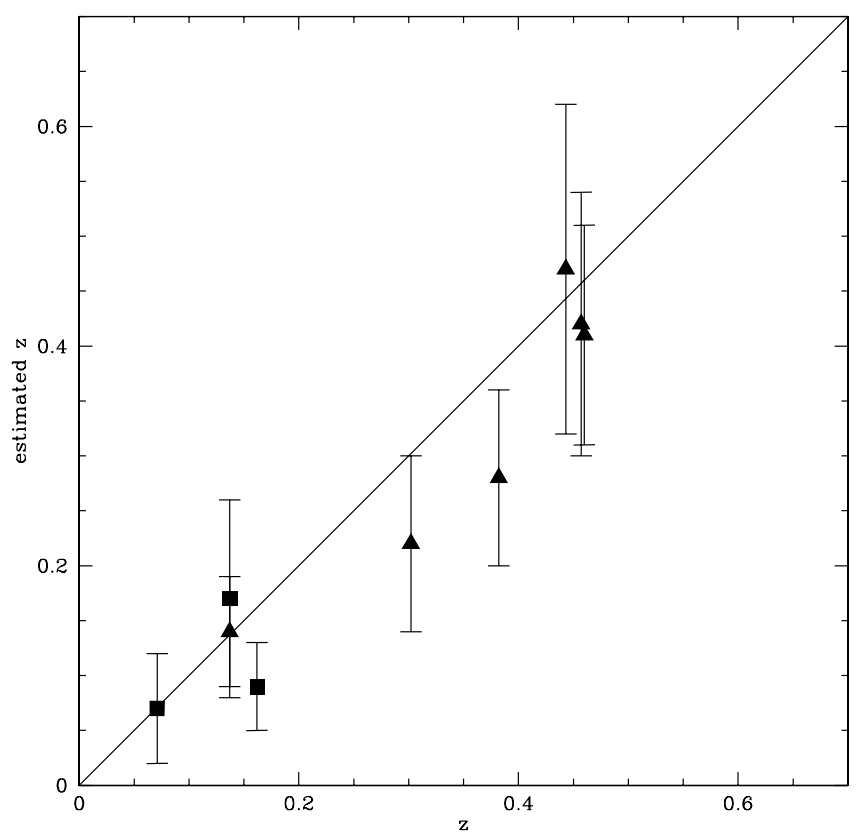

FIG. 10.-Comparison between the spectroscopic redshift $z$ and the value estimated from Ca II equivalent widths (see $\S 4.2 .4$ and Fig. 8). Triangles refer to objects observed with the ESO $3.6 \mathrm{~m}$ telescope and squares to objects observed with the ESO VLT (this work).
2133-449: This source was discovered because of its optical variability by Hawkins et al. (1991). Optical spectroscopy by Hawkins et al. (1991) and Heidt et al. (2004) led to completely featureless spectra. Our VLT observations clearly show the presence of an intervening absorption feature at $4250 \AA$, a tentative identification of which is intervening $\mathrm{Mg}$ II at $z=0.52$ (see Churchill et al. 2005). The lower limit on $z$ derived from $\mathrm{EW}_{\text {min }}$ is $z>0.98$.

2136-428: The spectrum obtained by Hawkins et al. (1991), who discovered this source in studying its optical variability, is completely featureless. Our VLT observations show several absorption features due to the ISM of our Galaxy: DIBs at 4428, 4726, 4882, and $5772 \AA$, Ca II $\lambda \lambda 3934,3968$, and $\mathrm{Na}$ I $\lambda 5892$ atomic lines. The feature at $5942 \AA$ could be $\mathrm{Ca}$ II $\lambda 3968$ at $z=0.497$. Since at this redshift the Ca II $\lambda 3934$ line should fall at $5890 \AA$, where it would be strongly contaminated by the interstellar $\mathrm{Na}$ I absorption, the redshift estimate is only tentative. The lower limit deduced by the minimum measurable equivalent width is $z>0.24$.

2214-313: Our VLT spectrum of this object clearly shows the typical spectral signature of the host galaxy (Ca II $\lambda \lambda 3934,3968$, and $G$ band 24305 ) at $z=0.46$. The best-fit decomposition gives $\alpha=0.9$ for the nonthermal component and $M_{R}=-22.3$ for the host. Previous optical spectroscopy performed by Bade et al. (1994) with the ESO $3.6 \mathrm{~m}$ telescope failed to detect any spectral feature.

2223-114: Optical observations of this radio source obtained by Véron-Cetty \& Véron (1993b) did not show any intrinsic spectral feature. In our spectrum, which extends further in the red, we detect a single narrow emission line at $7367 \AA(\mathrm{EW}=$ $5 \AA$ ). This is a real feature since it clearly appears on each of the three individual spectra (see $\S 3$ ). A possible identification of this line is [O II] $\lambda 3727$ at $z=0.977$, while $\mathrm{Mg}$ II $\lambda 2798$ gives $z=1.633$. We discard this second classification because the line FWHM (1200 $\left.\mathrm{km} \mathrm{s}^{-1}\right)$ is typical for a narrow line such as [O II], while for $\mathrm{Mg}$ II a larger value would be expected. Moreover, with a Mg II identification both C IV $\lambda 1549$ and C III] $\lambda 1909$ broad lines would be expected inside the observed spectral range, but no other features are detected.

2233 - 148: The redshift $z=0.325$ reported by Johnston et al. (1995) is due to confusion with the source HB89 2233+134 in Schmidt \& Green (1983). Drinkwater et al. (1997) report an intervening system at $z=0.609$ but without giving an identification of the corresponding absorption feature. We detect several absorption features in the spectrum. In particular, we propose to identify the features at 4165 and $4183 \AA$ as $\mathrm{Mg}$ II at $z=0.492$, while using the $\mathrm{EW}_{\min }$ estimate from the spectrum, $z>0.65$ is found.

2254-204: Previous optical spectroscopy (Véron-Cetty \& Véron 1993b; Hook et al. 2003) of this BL Lac object from the 1 Jy sample showed completely featureless spectra. With the VLT we are able to detect faint interstellar absorptions of $\mathrm{Ca}$ II $\lambda \lambda 3934,3968$ and $\mathrm{Na}$ I $\lambda 5892$, but no intrinsic or intervening spectral lines are found. The inferred redshift limit is $z>0.47$.

2307-375: This source was first classified as a BL Lac in the RSBC-NVSS sample (Bauer et al. 2000). The classification was then confirmed by the SS. No previous optical spectroscopy has been published. Our VLT spectrum is featureless, allowing us to set only a lower limit to the redshift of $z>1$.

2342-153: This source is part of the EMSS sample of BLLs. Our VLT data, as well as previous optical spectroscopy with the $6.5 \mathrm{~m}$ telescope of the MMT Observatory (Rector et al. 2000), showed a featureless spectrum. From $\mathrm{EW}_{\min }$ we derive $z>1$.

2354-021: This object was discussed in Paper I. Here we report only the spectrum, in Figure 2. 
2354-175: This X-ray source from the ROSAT All Sky Survey is classified as a BL Lac candidate in the RBSC-NVSS sample (Bauer et al. 2000) and in the SS. No previous spectroscopy was published in the literature. Our $\mathrm{S} / \mathrm{N}=150$ VLT spectrum is featureless, allowing only a lower limit of $z>0.85$ to the redshift.

\section{SUMMARY AND CONCLUSIONS}

Out of 42 objects observed we confirm the BL Lac classification for 36 sources, and for 18 of them we are able to measure/ confirm the redshift. This information allows us to derive the luminosity of the objects. The distribution in the $V$-band luminositydistance plane is indeed fully consistent with that observed for BLLs of known redshift in the combination of the Padovani \& Giommi (1995a) and the SS sample (see Fig. 11). We note that the sources in this combined list are affected by the typical selection effect of incomplete flux-limited samples; the envelope of the objects in fact follows the expected behavior for sources with constant $V$ magnitude, centered around $V=18$, with a spread of $\sim 3$ mag. The objects discussed here (filled circles and arrows) follow the same distribution, with absolute magnitudes ranging between -21.5 and -27.5 , slightly increasing with the redshift.

In 18 cases the optical spectra remain lineless in spite of the high $\mathrm{S} / \mathrm{N}$ of the obtained optical spectra. This indicates that if they are hosted by galaxies of standard luminosity they likely have very luminous or extremely beamed nuclei (see also Sbarufatti et al. 2005a). In the latter case one may expect to see the most extreme cases of relativistic beaming, making these sources ideal targets for milliarcsecond resolution radio observations. Alternatively, if the host galaxies were underluminous, these objects could be rare examples of dwarf galaxies hosting an AGN (see Sbarufatti et al. 2005a).

The high $\mathrm{S} / \mathrm{N}$ of most of the optical spectra obtained at VLT represents a frontier for the determination of the redshift of BLLs with current instrumentation, and further improvement of the issue will not be easy to get. In particular, the four brightest objects $(R<16$; 0048-099, 1553+113, 1722+119, and 2136-428) we observed at the VLT have values of $\mathrm{EW}_{\text {min }}$ smaller than $0.25 \AA$. These objects belong to an interesting subpopulation of BLLs

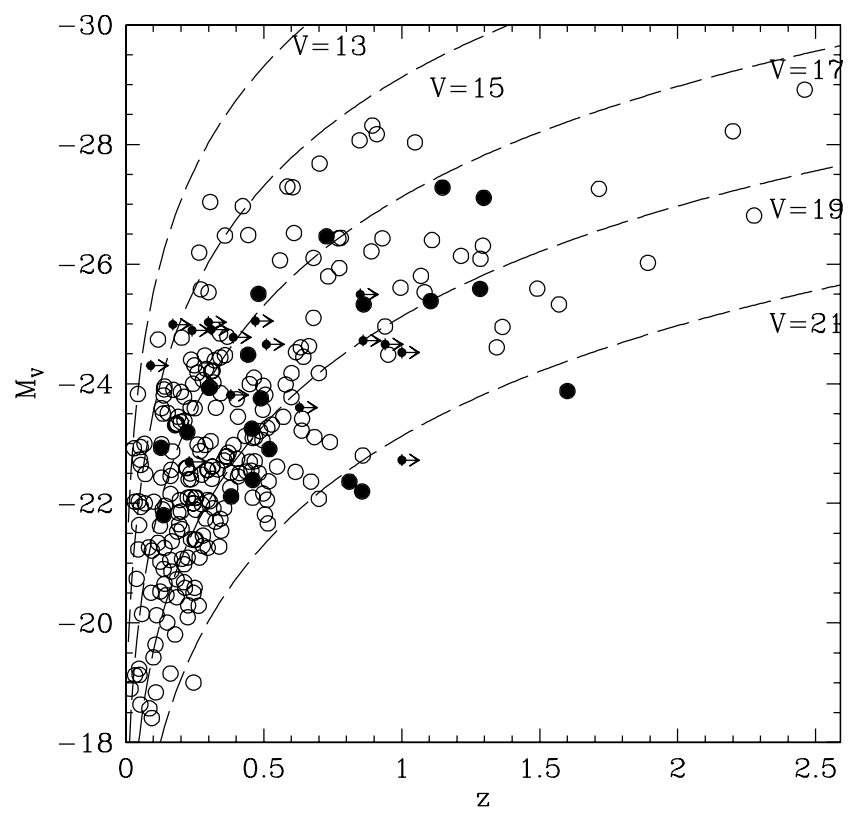

FIG. 11. $-M_{V}$ vs. redshift distribution of Padovani \& Giommi (1995a) + SS sample with redshifts known from literature (open circles) and BLLs, the redshifts of which have been determined in Paper I and in this work (filled circles), including redshift lower limits (arrows).

with extreme nuclear (and/or host) properties for which it is not actually possible to derive the intrinsic physical parameters. One possibility is to image the source when it is particularly faint in order to improve the detection of the host galaxy and to derive an imaging redshift (Sbarufatti et al. 2005a). Deep spectroscopic observations in the near-IR may also prove to be effective in the determination of the redshift, considering that this region of the spectrum is poorly known.

We would like to thank the referee, John Stocke, for his accurate and helpful comments, which allowed us to improve the quality of this work.
Allington-Smith, J. R., Peacock, J. A., \& Dunlop, J. S. 1991, MNRAS, 253, 287

Appenzeller, I., et al. 1998, Messenger, 94, 1

Arp, H. 1977, in IAU Colloq. 37, Decalages vers le Rouge et Expansion de L'Univers, ed. C. Balkowski \& B. E. Westerlund (Paris: Centre National de la Recherche Scientifique), 377

Bade, N., Fink, H. H., \& Engels, D. 1994, A\&A, 286, 381

Baldwin, J. A., Burbidge, E. M., Hazard, C., Murdoch, H. S., Robinson, L. B., \& Wampler, E. J. 1973, ApJ, 185, 739

Bauer, F. E., Condon, J. J., Thuan, T. X., \& Broderick, J. J. 2000, ApJS, 129, 547

Bressan, A., Granato, G. L., \& Silva, L. 1998, A\&A, 332, 135

Burbidge, G. 1996, A\&A, 309, 9

Burbidge, G., \& Hewitt, A. 1987, AJ, 93, 1

Carangelo, N., Falomo, R., Kotilainen, J., Treves, A., \& Ulrich, M.-H. 2003, A\&A, 412, 651

Cardelli, J. A., Clayton, G. C., \& Mathis, J. S. 1989, ApJ, 345, 245

Churchill, C. W., Kacprzac, G. G., \& Steidel, C. C. 2005, in IAU Colloq. 199, Probing Galaxies through Quasar Absorption Lines, ed. P. R. Williams, C.-G. Shu, \& B. Menard (Cambridge: Cambridge Univ. Press), 24

Condon, J. J., Cotton, W. D., Greisen, E. W., Yin, Q. F., Perley, R. A., Taylor, G. B., \& Broderick, J. J. 1998, AJ, 115, 1693

Croom, S. M., Smith, R. J., Boyle, B. J., Shanks, T., Miller, L., Outram, P. J., \& Loaring, N. S. 2004, MNRAS, 349, 1397

della Ceca, R., Palumbo, G. G. C., Persic, M., Boldt, E. A., Marshall, E. E., \& de Zotti, G. 1990, ApJS, 72, 471

Drinkwater, M. J., et al. 1997, MNRAS, 284, 85

\section{EFERENCES}

Ellison, S. L., Yan, L., Hook, I. M., Pettini, M., Wall, J. V., \& Shaver, P. 2001, A\&A, 379, 393

Falomo, R. 1996, MNRAS, 283, 241

Falomo, R., Bersanelli, M., Bouchet, P., \& Tanzi, E. G. 1993, AJ, 106, 11

Falomo, R., \& Kotilainen, J. K. 1999, A\&A, 352, 85

Falomo, R., Scarpa, R., \& Bersanelli, M. 1994, ApJS, 93, 125

Falomo, R., \& Treves, A. 1990, PASP, 102, 1120

Fricke, K. J., Kollatschny, W., \& Witzel, A. 1983, A\&A, 117, 60

Galazutdinov, G. A., Musaev, F. A., Krełowski, J., \& Walker, G. A. H. 2000, PASP, 112, 648

Gioia, I. M., Maccacaro, T., Schild, R. E., Wolter, A., Stocke, J. T., Morris, S. L., \& Henry, J. P. 1990, ApJS, 72, 567

Giommi, P., Menna, M. T., \& Padovani, P. 1999, MNRAS, 310, 465

Giommi, P., Piranomonte, S., Perri, M., \& Padovani, P. 2005, A\&A, 434, 385

Green, R. F., Schmidt, M., \& Liebert, J. 1986, ApJS, 61, 305

Griffiths, R. E., Wilson, A. S., Ward, M. J., Tapia, S., \& Ulvestad, J. S. 1989, MNRAS, 240, 33

Hawkins, M. R. S., Veron, P., Hunstead, R. W., \& Burgess, A. M. 1991, A\&A, 248,421

Hazard, C., \& Murdoch, H. S. 1977, Australian J. Phys. Astrophys. Suppl., 42, 1 Heidt, J., Nilsson, K., Sillanpää, A., Takalo, L. O., \& Pursimo, T. 1999, A\&A, 341,683

Heidt, J., Tröller, M., Nilsson, K., Jäger, K., Takalo, L., Rekola, R., \& Sillanpää, A. 2004, A\&A, 418, 813

Henriksen, M. J., Marshall, F. E., \& Mushotzky, R. F. 1984, ApJ, 284, 491

. 1993, ApJS, 87, 451 
Hook, I. M., Shaver, P. A., Jackson, C. A., Wall, J. V., \& Kellermann, K. I. 2003, A\&A, 399, 469

Horne, K. 1986, PASP, 98, 609

Jaunsen, A. O., Jablonski, M., Pettersen, B. R., \& Stabell, R. 1995, A\&A, 300, 323

Johnston, K. J., et al. 1995, AJ, 110, 880

Kinney, A. L., Calzetti, D., Bohlin, R. C., McQuade, K., Storchi-Bergmann, T., \& Schmitt, H. R. 1996, ApJ, 467, 38

Landt, H., Padovani, P., Perlman, E. S., Giommi, P., Bignall, H., \& Tzioumis, A. 2001, MNRAS, 323, 757

Laurent-Muehleisen, S. A., Kollgaard, R. I., Ciardullo, R., Feigelson, E. D., Brinkmann, W., \& Siebert, J. 1998, ApJS, 118, 127

Londish, D., et al. 2002, MNRAS, 334, 941

MacAlphine, G. M., \& Williams, G. A. 1981, ApJS, 45, 113

Marchã, M. J. M., Browne, I. W. A., Impey, C. D., \& Smith, P. S. 1996, MNRAS, 281, 425

Nilsson, K., Pursimo, T., Heidt, J., Takalo, L. O., Sillanpää, A., \& Brinkmann, W. 2003, A\&A, 400, 95

Oke, J. B. 1990, AJ, 99, 1621

Padovani, P., \& Giommi, P. 1995a, MNRAS, 277, 1477

. 1995b, ApJ, 444, 567

Perlman, E. S., et al. 1996, ApJS, 104, 251

Pettini, M., Smith, L. J., King, D. L., \& Hunstead, R. W. 1997, ApJ, 486, 665

Prochaska, J. X., et al. 2001, ApJS, 137, 21

Rector, T. A., \& Stocke, J. T. 2001, AJ, 122, 565

Rector, T. A., Stocke, J. T., Perlman, E. S., Morris, S. L., \& Gioia, I. M. 2000, AJ, 120, 1626

Rusk, R., \& Seaquist, E. R. 1985, AJ, 90, 30

Sbarufatti, B. 2005, Ph.D. thesis, Univ. Studi Milano-Bicocca

Sbarufatti, B., Treves, A., \& Falomo, R. 2005a, ApJ, 635, 173

Sbarufatti, B., Treves, A., Falomo, R., Heidt, J., Kotilainen, J., \& Scarpa, R. 2005b, AJ, 129, 559 (Paper I)

Schlegel, D. J., Finkbeiner, D. P., \& Davis, M. 1998, ApJ, 500, 525

Schmidt, G. D., Vennes, S., Wickramasinghe, D. T., \& Ferrario, L. 2001, MNRAS, 328, 203
Schmidt, G. D., et al. 2003, ApJ, 595, 1101

Schmidt, M., \& Green, R. F. 1983, ApJ, 269, 352

Sowards-Emmerd, D., Romani, R. W., Michelson, P. F., Healey, S. E., \& Nolan, P. L. 2005, ApJ, 626, 95

Stickel, M., Fried, J. W., Kühr, H., Padovani, P., \& Urry, C. M. 1991, ApJ, 374, 431

Stickel, M., \& Kühr, H. 1993, A\&AS, 100, 395

Sutton, J. M., Davies, I. M., Little, A. G., \& Murdoch, H. S. 1974, Australian J. Phys. Astrophys. Suppl., 33, 1

Thompson, D. J., \& Djorgovski, S. 1990, PASP, 102, 959

Tody, D. 1986, Proc. SPIE, 627, 733

1993, in ASP Conf. Ser. 52, Astronomical Data Analysis Software and Systems II, ed. R. J. Hanisch, R. J. V. Brissenden, \& J. Barnes (San Francisco: ASP), 173

Urry, C. M., Scarpa, R., O’Dowd, M., Falomo, R., Pesce, J. E., \& Treves, A. 2000, ApJ, 532, 816

Véron, P. 1996, A\&A, 310, 381

Véron, P., Véron-Cetty, M.-P., Djorgovski, S., Magain, P., Meylan, G., \& Surdej, J. 1990, A\&AS, 86, 543

Véron-Cetty, M.-P., \& Véron, P. 1993a, A Catalogue of Quasars and Active Nuclei (6th ed.; Garching: ESO)

. 1993b, A\&AS, 100, 521

2001, A\&A, 374, 92 2003, A\&A, 412, 399

Voges, W., et al. 1999, A\&A, 349, 389

Warren, S. J., Møller, P., Fall, S. M., \& Jakobsen, P. 2001, MNRAS, 326, 759

White, G. L., Jauncey, D. L., Wright, A. E., Batty, M. J., Savage, A., Peterson, B. A., \& Gulkis, S. 1988, ApJ, 327, 561

White, N. E., Giommi, P., \& Angelini, L. 1994, IAU Circ., 6100, 1

Wills, D., \& Wills, B. J. 1976, ApJS, 31, 143

Wisotzki, L. 2000, A\&A, 353, 861

Woo, J.-H., Urry, M. C., van der Marel, R. P., Lira, P., \& Maza, J. 2005, ApJ, 631,762

Wurtz, R., Stocke, J. T., \& Yee, H. K. C. 1996, ApJS, 103, 109

Zotov, N. V., \& Tapia, S. 1979, ApJ, 229, L5 